\title{
A Modal Proof Theory for Polynomial Coalgebras
}

by

\section{David Friggens}

\author{
A thesis \\ submitted to Victoria University of Wellington \\ in fulfilment of the \\ requirements for the degree of \\ Master of Science \\ in Logic and Computation. \\ Victoria University of Wellington \\ 2004
}




\begin{abstract}
The abstract mathematical structures known as coalgebras are of increasing interest in computer science for their use in modelling certain types of data structures and programs. Traditional algebraic methods describe objects in terms of their construction, whilst coalgebraic methods describe objects in terms of their decomposition, or observational behaviour. The latter techniques are particularly useful for modelling infinite data structures and providing semantics for object-oriented programming languages, such as Java.

There have been many different logics developed for reasoning about coalgebras of particular functors, most involving modal logic. We define a modal logic for coalgebras of polynomial functors, extending Rößiger's logic [33], whose proof theory was limited to using finite constant sets, by adding an operator from Goldblatt [11]. From the semantics we define a canonical coalgebra that provides a natural construction of a final coalgebra for the relevant functor. We then give an infinitary axiomatization and syntactic proof relation that is sound and complete for functors constructed from countable constant sets.
\end{abstract}




\section{Acknowledgments}

I am deeply indebted to my supervisor, Professor Robert Goldblatt, for pointing me in the right direction and keeping my wheels on the tracks. His mathematical advice is the best anyone could hope for.

I would like to thank Ranald Clouston for many discussions on logic and life in general. This thesis (and my life in general) are the better for them.

I would like to thank all the people at the Centre for Logic, Language and Computation at Victoria who have taught me through my undergraduate years for introducing me to the exciting world of logic.

Financially, I have been supported by a scholarship from the Logic and Computation programme of the New Zealand Institute for Mathematics and its Applications. I am grateful for the hospitality of the Institute for Information Sciences and Technology at Massey University.

This thesis would have been a nightmare to produce manually-I am very grateful for the work of Donald Knuth, Leslie Lamport and many others in producing the typesetting system $\mathrm{LT}_{\mathrm{E}} \mathrm{X}$. My files were compiled thanks to Thomas Esser's teTEX distribution; I conform to VUW conventions thanks to Neil Leslie's vuwthes is package; my proof boxes are in the right place thanks to Paul Taylor's QED package; and my diagrams are just how I want them thanks to the excellent package $X_{Y}$-pic by Kristoffer Rose and Ross Moore.

Finally, I owe more than I can say to Olivia and my family for their love and support. I appreciate it much more than it shows. 


\section{Contents}

\begin{tabular}{|ll} 
Acknowledgments & i
\end{tabular}

\begin{tabular}{lll}
\hline & Introduction & $\mathbf{1}$
\end{tabular}

1.1 From algebras to systems . . . . . . . . . . . . . . . . 1

1.2 Relations between systems . . . . . . . . . . . . . . 4

1.3 Definitions and proofs: (co)induction . . . . . . . . . . . 6

1.4 Specification language: coalgebraic logics . . . . . . . . . . 11

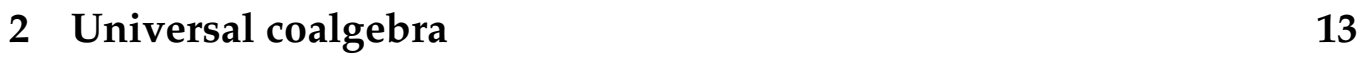

2.1 Set Notation . . . . . . . . . . . . . . . . . . . 13

2.2 Polynomial Functors . . . . . . . . . . . . . . . . . 14

2.3 Universal Coalgebra $\ldots \ldots \ldots \ldots$

2.3 .1 Bisimulation . . . . . . . . . . . . . . . 24

2.3 .2 Final coalgebra . . . . . . . . . . . . . . . . . . . . . . . . . 30

2.4 Paths . . . . . . . . . . . . . . . . . . . . 32

3 Syntax and Semantics of Formulas 39

4 Axiomatic Construction 44

\begin{tabular}{|lll}
5 & Canonical coalgebras & 48
\end{tabular}

$\begin{array}{lll}6 & \text { Proof Theory } & 55\end{array}$ 


\section{Chapter 1}

\section{Introduction}

Traditionally formal methods in computer science have used algebraic methods for describing data structures and programs. However there has recently been increasing interest in the use of coalgebraic methods, as they are able to be used in cases where algebraic methods are not. Algebra is a well-established area of mathematics and can be studied abstractly within universal algebra, and at an even more abstract level within category theory (see e.g. Mac Lane [26]). The notion of a coalgebra comes from category theory, as the formal dual of an algebra. As such, many algebraic concepts and results have dual coalgebraic counterparts - e.g. congruence and bisimulation equivalence, initial algebra and final coalgebra, induction and coinduction. These will be explored in further detail below.

\subsection{From algebras to systems}

The main conceptual difference between algebraic and coalgebraic methods is that the former represent objects in terms of their construction operations. In contrast coalgebraic methods represent objects in terms of the way in which they are decomposed, or observed to behave and change - using their destructor operations (also called observers, mutators, accessors 
or transition maps).

To illustrate these concepts consider the case of (finite) lists, $A^{*}$, from a set $A$. A list is either the empty list, $\varepsilon$, or the concatenation of an element, $a \in A$, and a list $l$, written $a \cdot l$. Using the singleton set $1=\{\star\}$ we can combine the construction functions

$$
\begin{array}{rrr}
\text { empty : } & 1 \longrightarrow A^{*}: \quad \star \quad \longmapsto \varepsilon \\
\text { cons : } & A \times A^{*} \longrightarrow A^{*}: \quad\langle a, l\rangle \longmapsto a \cdot l
\end{array}
$$

into a single entity that we call an algebra:

$$
\left\langle A^{*}, \alpha_{l}:\left(1+\left(A \times A^{*}\right)\right) \longrightarrow A^{*}\right\rangle
$$

where the function $\alpha_{l}$ constructs a list object from the singleton or an element and list.

To compare, consider the case of streams (or infinite lists), $A^{\omega}$, from $A$. Streams have no construction functions, but can be observed through their decomposition functions:

$$
\begin{aligned}
& \text { head }: A^{\omega} \longrightarrow A: a \cdot l \longrightarrow a \\
& \text { tail : } A^{\omega} \longrightarrow A^{\omega}: a \cdot l \longrightarrow l
\end{aligned}
$$

We can combine these in a similar fashion to form a structure we call a coalgebra:

$$
\left\langle A^{\omega}, \alpha_{s}: A^{\omega} \longrightarrow\left(A \times A^{\omega}\right)\right\rangle
$$

where the function $\alpha_{s}$ decomposes a stream in to an element and another stream.

Similarly, the set of finite and infinite lists from $A, A^{\infty}=A^{*} \cup A^{\omega}$, has no construction functions. Its decomposition functions include the same head and tail as before, with the addition of the empty list observation function:

$$
\begin{array}{r}
\text { null }: A^{\infty} \longrightarrow 1: \quad \varepsilon \longmapsto \star \\
\text { head }: A^{\infty} \longrightarrow A: a \cdot l \longmapsto a \\
\text { tail }: A^{\infty} \longrightarrow A^{\infty}: a \cdot l \longmapsto l
\end{array}
$$


These functions combine to form a coalgebra

$$
\left\langle A^{\infty}, \quad \alpha_{i}: A^{\infty} \longrightarrow\left(1+\left(A \times A^{\infty}\right)\right)\right\rangle
$$

where the function $\alpha_{i}$ decomposes a list as the singleton or an element and another (possibly infinite) list.

We have noted that the latter two examples have decomposition functions and no construction functions, and that the first example does have construction functions. Using the same reasoning as above, we can see that the case of lists $A^{*}$ has decomposition functions too - in fact they are the same as for $A^{\infty}$ : null, head and tail. Hence we can also form a coalgebra for $A^{*}$ :

$$
\left\langle A^{*}, \quad \alpha_{l}^{\prime}: A^{*} \longrightarrow\left(1+\left(A \times A^{*}\right)\right)\right\rangle
$$

It is useful to view coalgebras generically as state based systems, with a state space, $A$, and a transition structure, $\alpha: A \longrightarrow T A$, where $T$ is some operation on the state space. $T$ is known as a functor and is a functorial operation, in that it acts on functions between sets as well as on sets (i.e. if $f: B \longrightarrow C$ is a function then so is $T f: T B \longrightarrow T C)$. Formal definitions will be given in Chapter 2, but for now consider this informal definition from Pattinson [30] that a state based system meets the following conditions.

(1) The behaviour of the system depends on an internal state, which is invisible to the user of the system.

(2) The system is reactive, i.e. not necessarily terminating, and interacts with its environment.

(3) The system comes with a set of operations, through which this interaction takes place.

The systems that we consider use polynomial functors, which correspond to deterministic systems with various combinations of input and output, and cover most applications in computer science. They are of the form 


\begin{tabular}{lcl} 
Name & Symbol & System actions \\
\hline Identity & Id & state transition \\
Constant & $\bar{D}$ & output from set $D$ \\
Power & $T^{D}$ & input from set $D$ before $T$ \\
Product & $T_{1} \times T_{2}$ & $T_{1}$ "and" $T_{2}$ \\
Coproduct & $T_{1}+T_{2}$ & $T_{1}$ "or" $T_{2}$
\end{tabular}

In computer science, common situations where coalgebraic methods are necessary include infinite data structures (e.g. streams and non-wellfounded sets [2]) and dynamic black-box systems, where the state internals are hidden from the user (e.g. the object-oriented programming language paradigm). An important and currently very active use of coalgebras in computer science at present is in the formulation of semantics for object-oriented programs, especially in Java [32, 17, 31, 19, 20, 25].

\subsection{Relations between systems}

In universal coalgebra there are two main ways of describing the relationship between coalgebras. The first is via (homo)morphisms, which transform one coalgebra to another coalgebra of the same functor. As with morphisms of algebras, morphisms of coalgebras are intuitively structure preserving functions.

The second relationship between coalgebras is bisimulation, or behavioural equivalence. Since we are dealing with black-box systems the closest measure we can have of equivalence between systems is of indistinguishable observable behaviour. A bisimulation between coalgebras is intuitively a structure preserving relation. It is dual to the algebraic notion of a substitution relation and relates states that are observationally indistinguishable, or bisimilar. Similarly, a bisimulation equivalence - a bisimulation that is also an equivalence relation - is dual to the algebraic 
notion of congruence. To illustrate, consider the following systems.

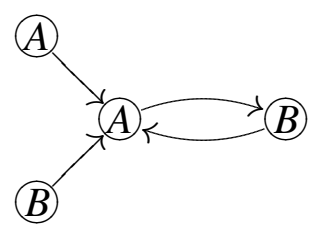

In this system the two states labelled $B$ are behaviourally indistinguishable - they both move to an $A$ state - whilst the states labelled $A$ are distinguishable - one moves to an $A$ state and the other to a $B$ state. The next two pairs of systems are indistinguishable from one another, in the sense that for each state there is a state of the other that can simulate its observable behaviour.
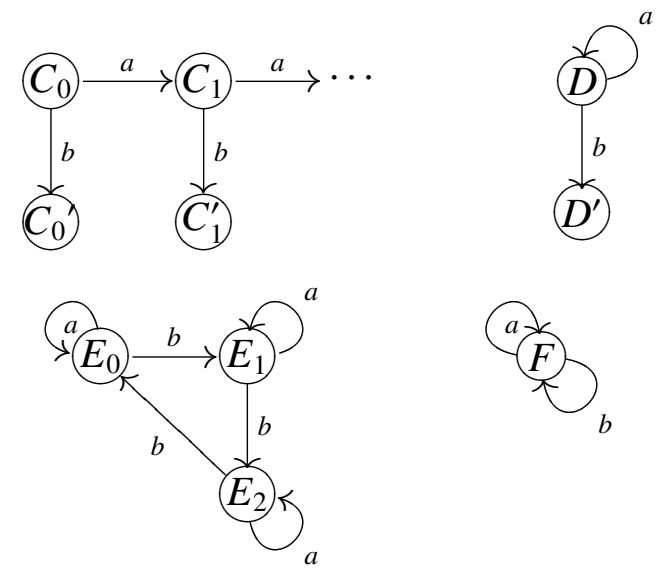

Bisimulation equivalence is an important notion for the following two reasons:

(1) if two states are bisimilar then one may replace the other without affecting the behaviour of the system; and

(2) when making assertions about a system we want to refer only to its observable behaviour and not constrain its internal representation.

The notion of bisimulation comes via concurrency theory. It first appeared in [29] as a relation of mutual simulation between states of two automata; it was shown that deterministic automata related by bisimulation 
accept the same input. The categorial definition we use (Definition 2.12) is due to Aczel and Mendler [1]. The concept of bisimilar states (or concurrent processes) satisfying the same formulas of a logical language (Theorem 3.6) is due to Hennessy and Milner [13, 14].

\subsection{Definitions and proofs: (co)induction}

In universal algebra the principle of induction is used for definitions and proofs of algebraic objects. It uses initial algebras, which have a unique morphism to any other algebra. There is a dual notion of a final coalgebra, which has a unique morphism from any other coalgebra:

$$
\begin{gathered}
\left(\begin{array}{c}
\text { initial } \\
\text { algebra }
\end{array}\right) \underset{\text { morphism }}{\text { unique }}>\left(\begin{array}{c}
\text { arbitrary } \\
\text { algebra }
\end{array}\right) \\
\left(\begin{array}{c}
\text { arbitrary } \\
\text { coalgebra }
\end{array}\right) \underset{\text { morphism }}{\text { unique }}>\left(\begin{array}{c}
\text { final } \\
\text { coalgebra }
\end{array}\right)
\end{gathered}
$$

Final coalgebras are used in definitions and proofs by coinduction. In rough terms, for both induction and coinduction, a definition involves establishing the existence of the morphism above, and a proof involves establishing its uniqueness.

For an inductive definition of a function $f$, we define the value of $f$ on all constructor functions. Recall the example of lists $A^{*}$, with constructors empty and cons. We would define the functions len, which gives the length of lists, and double, which replaces each element in the list with two copies, like sol?

$$
\begin{aligned}
\operatorname{len}(\operatorname{empty}(\star)) & =0 \\
\operatorname{len}(\operatorname{cons}(a, l)) & =1+\operatorname{len}(l)
\end{aligned}
$$

\footnotetext{
${ }^{1}$ The following examples of definition and proof by (co)induction are taken from Jacobs and Rutten [21].
} 


$$
\begin{aligned}
\text { double }(\operatorname{empty}(\star)) & =\varepsilon \\
\text { double }(\operatorname{cons}(a, l)) & =\operatorname{cons}(a, \operatorname{cons}(a, \text { double }(l)))
\end{aligned}
$$

Now suppose we want to prove that the length of double $(l)$ is twice that of $l$, i.e. $\operatorname{len}(\operatorname{double}(l))=2 \cdot \operatorname{len}(l)$. The ordinary way we would prove this is as follows:

$$
\begin{aligned}
& \operatorname{len}(\operatorname{double}(\operatorname{empty}(\star))) \\
= & \operatorname{len}(\operatorname{empty}(\star)) \\
= & 0 \\
= & 2 \cdot 0 \\
= & 2 \cdot \operatorname{len}(\operatorname{empty}(\star)) \\
& \text { len }(\operatorname{double}(\operatorname{cons}(a, l))) \\
= & \operatorname{len}(\operatorname{cons}(a, \operatorname{cons}(a, \operatorname{double}(l)))) \\
= & 1+1+\operatorname{len}(\operatorname{double}(l)) \\
\text { IH } & 2+2 \cdot \operatorname{len}(l) \\
= & 2 \cdot(1+\operatorname{len}(l)) \\
= & 2 \cdot \operatorname{len}(\operatorname{cons}(a, l))
\end{aligned}
$$

However it is possible to prove this in a more abstract way using the initial algebra, which we shall outline. It can be shown that the list algebra $\left\langle A^{*}, \alpha_{l}\right\rangle$ is an initial algebra for the functor $T(X)=1+A \times X$. This fact will allow us to prove the equality of the functions len $\circ$ double and $2 \cdot(-) \circ$ len. First, let us rearrange our definitions in a diagrammatic form:

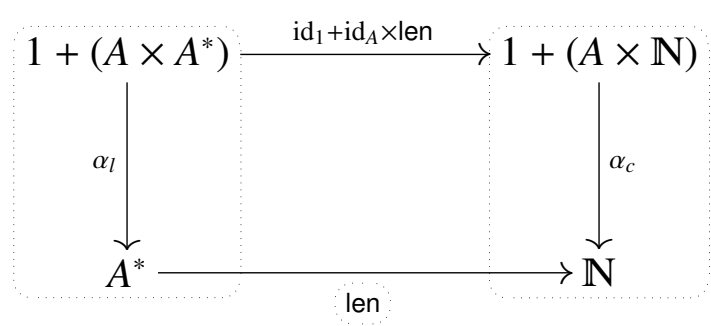




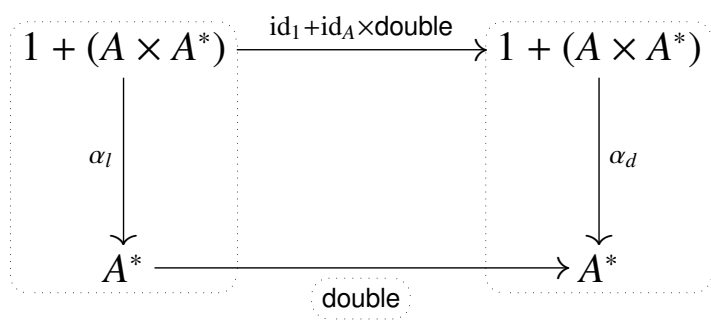

where

$$
\begin{aligned}
& \alpha_{l}(x)= \begin{cases}\operatorname{empty}(x) & \text { if } x=\star \\
\operatorname{cons}(a, l) & \text { if } x=\langle a, l\rangle\end{cases} \\
& \alpha_{c}(x)= \begin{cases}0 & \text { if } x=\star \\
1+n & \text { if } x=\langle a, n\rangle\end{cases} \\
& \alpha_{d}(x)= \begin{cases}(x) & \text { if } x=\star \\
\operatorname{cons}(a, \operatorname{cons}(a, l)) & \text { if } x=\langle a, l\rangle\end{cases}
\end{aligned}
$$

We see that len and double are structure preserving functions and are intuitively defined as the unique morphisms from the initial algebra $\left\langle A^{*}, \alpha_{l}\right\rangle$ to the algebras $\left\langle\mathbb{N}, \alpha_{c}\right\rangle$ and $\left\langle A^{*}, \alpha_{d}\right\rangle$, respectively. A similar approach shows that both of the functions len $\circ$ double and $2 \cdot(-) \circ$ len are morphisms from $\left\langle A^{*}, \alpha_{l}\right\rangle$ to the algebra $\left\langle\mathbb{N}, \alpha_{p}: 1+A \times \mathbb{N} \longrightarrow \mathbb{N}\right\rangle$, where

$$
\alpha_{p}(x)= \begin{cases}0 & \text { if } x=\star \\ 2+n & \text { if } x=\langle a, n\rangle\end{cases}
$$

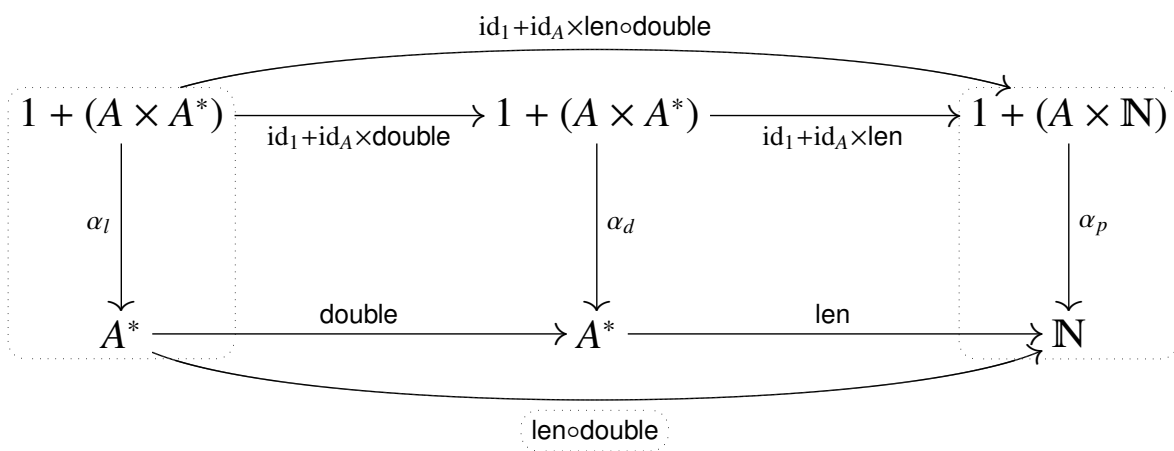




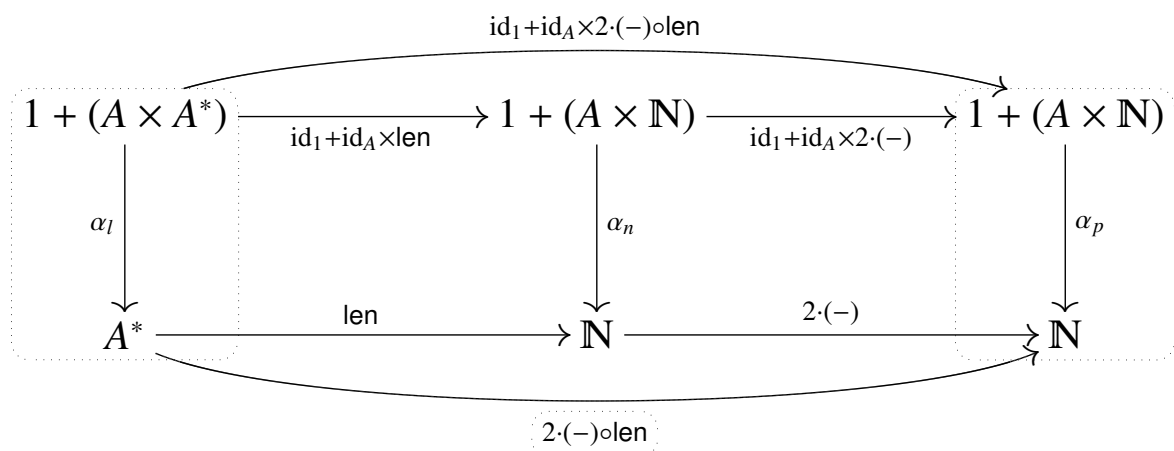

But $\left\langle A^{*}, \alpha_{l}\right\rangle$ is an initial algebra, so there is a unique morphism $f=$ len $\circ$ double $=2 \cdot(-) \circ$ len from $\left\langle A^{*}, \alpha_{l}\right\rangle$ to $\left\langle\mathbb{N}, \alpha_{p}\right\rangle$.

Dually, for a coinductive definition of a function $g$, we define the values of all decomposition functions on each outcome $g(x)$. Recall the example of streams $A^{\omega}$, with destructors head and tail. We would define the functions odd, which removes all elements from a stream in the even numbered positions, even, which removes all elements from a stream in the odd numbered positions, and merge, which combines two streams one element at a time to form a new stream, like so:

$$
\begin{aligned}
\operatorname{head}(\operatorname{odd}(l)) & =\operatorname{head}(l) \\
\operatorname{tail}(\operatorname{odd}(l)) & =\operatorname{odd}(\operatorname{tail}(\operatorname{tail}(l))) \\
\operatorname{even}= & \operatorname{odd} \circ \text { tail } \\
\operatorname{head}\left(\operatorname{merge}\left(l_{1}, l_{2}\right)\right) & =\operatorname{head}\left(l_{1}\right) \\
\operatorname{tail}\left(\operatorname{merge}\left(l_{1}, l_{2}\right)\right) & =\operatorname{merge}\left(l_{2}, \operatorname{tail}\left(l_{1}\right)\right)
\end{aligned}
$$

Now suppose we want to prove that merge $(\operatorname{odd}(l)$, even $(l))=l$. It can be shown that the stream coalgebra $\left\langle A^{\omega}, \alpha_{s}\right\rangle$ is a final coalgebra for the functor $T(X)=A \times X$. This fact will allow us to prove the equality of the function merge $\circ\langle$ odd, even〉 and the identity function. Let us rearrange our coin- 
ductive definitions in a diagrammatic form:
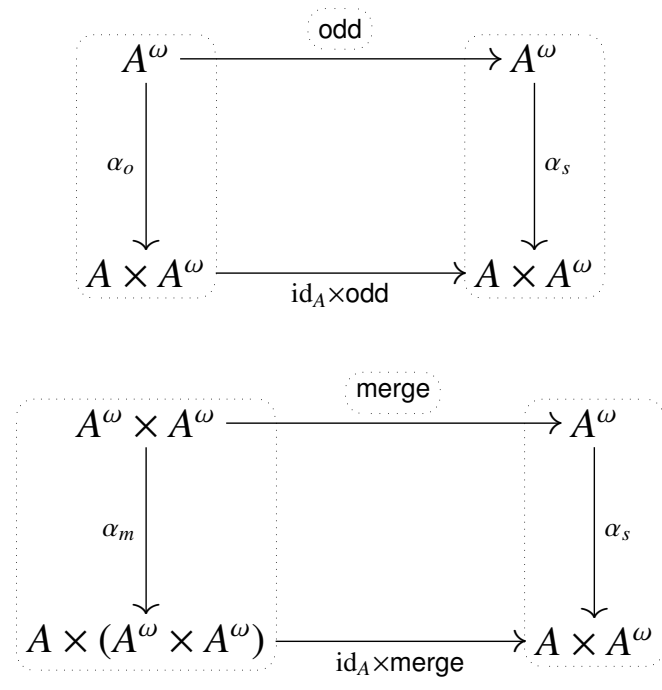

where $\alpha_{s}(l)=\langle\operatorname{head}(l)$, tail $(l)\rangle, \alpha_{o}(l)=\langle\operatorname{head}(l)$, tail(tail $\left.(l))\right\rangle$ and $\alpha_{m}\left(l_{1}, l_{2}\right)=$ $\left\langle\right.$ head $\left(l_{1}\right),\left\langle l_{2}\right.$, tail $\left.\left.\left(l_{1}\right)\right\rangle\right\rangle$. Similarly, but dually, to the inductive definitions, we see that odd and merge are structure preserving functions and are coinductively defined as the unique morphisms from the coalgebras $\left\langle A^{\omega}, \alpha_{o}\right\rangle$ and $\left\langle A^{\omega} \times A^{\omega}, \alpha_{m}\right\rangle$, respectively, to the final coalgebra $\left\langle A^{\omega}, \alpha_{s}\right\rangle$. To prove that $\operatorname{merge}(\operatorname{odd}(l)$, even $(l))=l$ we can show that merge $\circ\langle$ odd, even $\rangle$ is a morphism from $\left\langle A^{\omega}, \alpha_{s}\right\rangle$ to $\left\langle A^{\omega}, \alpha_{s}\right\rangle$. Thus, since $\left\langle A^{\omega}, \alpha_{s}\right\rangle$ is a final coalgebra and the identity function id $: A^{\omega} \longrightarrow A^{\omega}$ is trivially the unique morphism from $\left\langle A^{\omega}, \alpha_{s}\right\rangle$ to $\left\langle A^{\omega}, \alpha_{s}\right\rangle$, it follows that merge $\circ\langle$ odd, even $\rangle=$ id. To show that it is a morphism the following diagram must commute.

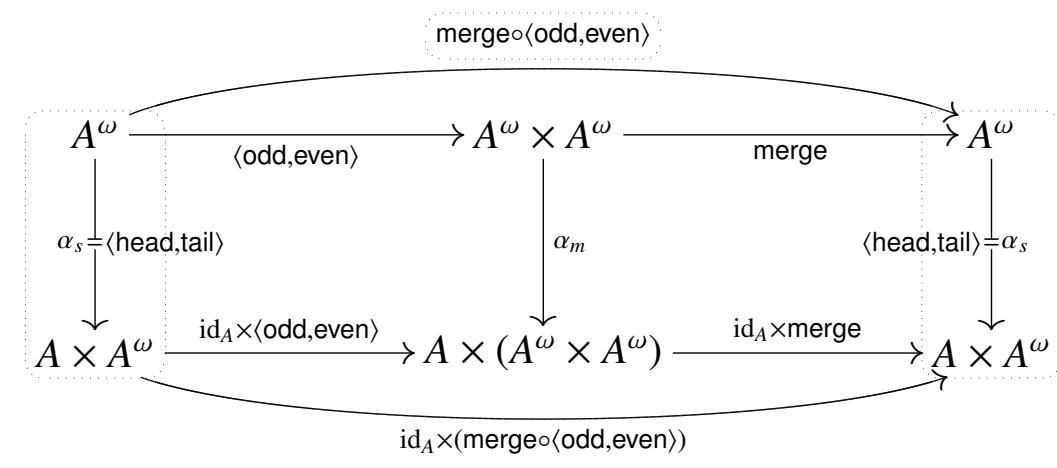


In other words, we must show that

$\langle$ head, tail $\rangle($ merge $\circ\langle$ odd, even $\rangle)=($ id $\times($ merge $\circ\langle$ odd, even $\rangle) \circ\langle$ head, tail $\rangle$

which follows from the following computations.

$$
\begin{aligned}
& \operatorname{head}(\operatorname{merge}(\operatorname{odd}(l), \operatorname{even}(l))) \\
= & \operatorname{head}(\operatorname{odd}(l)) \\
= & \operatorname{head}(l) \\
& \operatorname{tail}(\operatorname{merge}(\operatorname{odd}(l), \operatorname{even}(l))) \\
= & \operatorname{merge}(\operatorname{even}(l), \operatorname{tail}(\operatorname{odd}(l))) \\
= & \operatorname{merge}(\operatorname{even}(l), \operatorname{odd}(\operatorname{tail}(\operatorname{tail}(l)))) \\
= & \operatorname{merge}(\operatorname{odd}(\operatorname{tail}(l)), \operatorname{even}(\operatorname{tail}(l))) \\
= & (\operatorname{merge} \circ\langle\operatorname{odd}, \text { even }\rangle)(\operatorname{tail}(l))
\end{aligned}
$$

\subsection{Specification language: coalgebraic logics}

If a coalgebra is a system then its logic is the system's specification language. Equational logics for algebra have been extensively studied over many decades, but the study of coalgebras is much younger and there remains no definitive approach to logics for coalgebras. The appropriate logic for coalgebras is modal logic, and Kurz [22] has shown that it can be thought of as 'dual' to equational logic, when interpreted in a categorial context. Moss [27] was the first to use modal logic, introducing an infinitary modal logic for general functors, though it lacked a complete axiomatization. Corradini [5, 6], Cîrstea [3, 4] and Goldblatt [10] have published equational logics for monomial functors (polynomial without coproduct). Kurz [23] defined a finitary modal logic for polynomial functors of the form $\prod_{i=1}^{n}\left(\overline{B_{i}}+\overline{C_{i}} \times \mathrm{Id}\right)^{A_{i}}$, and Rößiger [33] defined another finitary modal logic for all polynomial functors. The 'syntax trees' of functors used in the latter restrict the proof theory to functors constructed from finite constant sets. Goldblatt [11] defined an equational logic for polynomial functors 
that is implicitly modal and is shown to subsume Rößiger's logic; it has no proof theory developed as yet.

We introduce a Hennessy-Milner style modal logic, the models of which are polynomial coalgebras of a fixed functor $T$. It extends the logic of Rößiger - instead of syntax trees we use the notion of paths, introduced in [18], to characterize the structure of functors and to examine the observational behaviour (output) of systems. Using a characterization of bisimulation through paths we show that logical equivalence of states coincides with bisimilarity. We define an axiomatization and proof theory, which is sound and complete if $T$ has constant sets of denumerable cardinality. Completeness is shown by a Henkin proof [7, 8], which involves the construction of a canonical model (coalgebra), where states are sets of formulas that satisfy all, and only, the formulas they contain. This canonical coalgebra, which can be constructed independent of the proof theory, is shown to be a final coalgebra for $T$. 


\section{Chapter 2}

\section{Universal coalgebra and paths for polynomial functors}

In this section we provide an exposition of universal coalgebra for polynomial functors over the category of sets, and paths between functors. We closely follow Rutten [34] for universal coalgebra and Goldblatt [11] for paths.

\subsection{Set Notation}

We briefly review some notions from set theory and the notation used here.

A function $f$ from set $A$ to set $B$ is denoted $f: A \longrightarrow B$, a partial function $g$ from $A$ to $B$ is denoted $g: A \circ B$ and a function $h$ mapping an element $a \in A$ to an element $b \in B$ is denoted $h: a \longmapsto b$.

The identity function on a set $A$ is denoted $\operatorname{id}_{A}: A \longrightarrow A$.

For sets $A$ and $D$ the $D$-th power of $A$ is the set of functions $A^{D}=$ $\{f \mid D \longrightarrow A\}$. For every $d \in D$ there is an evaluation function $e v_{d}: A^{D} \longrightarrow A$ where $e v_{d}(f)=f(d)$.

The product of sets is $A_{1} \times A_{2}=\left\{\left\langle a_{1}, a_{2}\right\rangle \mid a_{1} \in A_{1}, a_{2} \in A_{2}\right\}$. For $j \in\{1,2\}$ there are the projection functions $\pi_{j}: A_{1} \times A_{2} \longrightarrow A_{j}$. 
The coproduct (or disjoint union) of sets is $A_{1}+A_{2}=\left\{\left\langle 1, a_{1}\right\rangle \mid a_{1} \in A_{1}\right\} \cup$ $\left\{\left\langle 2, a_{2}\right\rangle \mid a_{2} \in A_{2}\right\}$. There are the injection functions $\iota_{1}: A_{1} \longrightarrow A_{1}+A_{2}: a_{1} \longmapsto$ $\left\langle 1, a_{1}\right\rangle$ and $\iota_{2}: A_{2} \longrightarrow A_{1}+A_{2}: a_{2} \longmapsto\left\langle 2, a_{2}\right\rangle$ and their (partial) inverses, the extraction functions $\varepsilon_{j}: A_{1}+A_{2} \multimap A_{j}:\left\langle j, a_{j}\right\rangle \longmapsto a_{j}$.

The powerset of $A$, the set of all subsets is $2^{A}=\{X \mid X \subseteq A\}$.

A relation $R$ between sets $A$ and $B$ is a subset of the product, i.e. $R \subseteq$ $A \times B$. When $\langle a, b\rangle \in R$ we often write $a R b$.

We say that a set $X \subseteq A$ is closed under a relation of the form $R \subseteq 2^{A} \times A$ if, for every $\langle Y, a\rangle \in R, Y \subseteq X$ implies $a \in X$.

We adopt the convention that whenever we write " $f(x) R g(y)$ " for any relation $R$ and partial functions $f$ and $g$ we mean $x \in \operatorname{Dom} f$ iff $y \in \operatorname{Dom} g$ and if both $f(x)$ and $g(y)$ are defined then $\langle f(x), g(y)\rangle \in R$. In particular, note that we use this convention for the relation " $=$ ".

\subsection{Polynomial Functors}

Definition 2.1 A functor on sets, $T:$ Set $\longrightarrow$ Set, is a function that maps

(1) every set $A$ to another set $T A$; and

(2) every function $f: A \longrightarrow B$ to another function $T f: T A \longrightarrow T B$

such that

(1) $T \mathrm{id}_{A}=\mathrm{id}_{T A} ;$ and

(2) $T(g \circ f)=(T g) \circ(T f)$.

DEFINITION 2.2 A functor $T$ is polynomial if it is finitely constructed from the following functors:

- the identity functor $\operatorname{Id}$, with $\operatorname{Id} A=A$ on sets $A$ and $\operatorname{Id} f=f$ on functions $f: A \longrightarrow B$ 
- for a fixed set $D \neq \varnothing$, the constant functor $\bar{D}$, with $\bar{D} A=D$ and $\bar{D} f=$ $\mathrm{id}_{D}$

- the $D$-th power functor $T^{D}$ of a functor $T$, with

$$
T^{D} A=(T A)^{D}
$$

and

$$
T^{D}(f):(T A)^{D} \longrightarrow(T B)^{D}: g \longmapsto T f \circ g
$$

- the product of two functors $T_{1} \times T_{2}$, with

$$
\left(T_{1} \times T_{2}\right)(A)=T_{1}(A) \times T_{2}(A)
$$

and

$$
\begin{aligned}
\left(T_{1} \times T_{2}\right)(f)=T_{1}(f) \times T_{2}(f): & T_{1} A \times T_{2} A \longrightarrow T_{1} B \times T_{2} B \\
: & \left\langle a_{1}, a_{2}\right\rangle \longmapsto\left\langle T_{1}(f)\left(a_{1}\right), T_{2}(f)\left(a_{2}\right)\right\rangle
\end{aligned}
$$

- the coproduct of two functors $T_{1}+T_{2}$, with

$$
\left(T_{1}+T_{2}\right)(A)=T_{1}(A)+T_{2}(A)
$$

and

$$
\begin{aligned}
\left(T_{1}+T_{2}\right)(f)=T_{1}(f)+T_{2}(f): & T_{1} A+T_{2} A \longrightarrow T_{1} B+T_{2} B \\
& : \iota_{j}(a) \longmapsto \iota_{j}\left(T_{j}(f)(a)\right)
\end{aligned}
$$

A functor $T_{1}$ used in the construction of a functor $T_{2}$ is known as a component of $T_{2}$.

From this point forward we shall assume that all functors are polynomial. We shall only use parentheses for clarification where necessary and will assume that product binds more tightly than coproduct and power more tightly than both, i.e. $T_{1}+\left(T_{2} \times T_{3}\right)=T_{1}+T_{2} \times T_{3} \neq\left(T_{1}+T_{2}\right) \times T_{3}$ and $T_{1} \times\left(T_{2}{ }^{D}\right)=T_{1} \times T_{2}{ }^{D} \neq\left(T_{1} \times T_{2}\right)^{D}$. Note that this convention includes dropping parentheses on application, i.e. $T_{1}+T_{2} f=\left(T_{1}+T_{2}\right) f$. 
EXAMPLE 2.3 Some examples of polynomial functors:

- As seen in the introduction a finite list is either the empty list or the pairing of an element of the constant set $A$ and another list. The construction and decomposition of lists is $T(X)=1+A \times X$, so the functor is $T=\overline{1}+\bar{A} \times$ Id.

- Streams decompose as an element and another stream, so the functor is $T=\bar{A} \times$ Id.

- Mealy machines are finite automata that require user input for state transitions and give output at each state. At each state in $X$ input from the constant set $I$ invokes a transition to another state in $X$ and output from the constant set $O$. The transition can be written as $T(X)=(O \times X)^{I}$ so the functor is $T=(\bar{O} \times \mathrm{Id})^{I}$.

\subsection{Universal Coalgebra}

DEFINITION 2.4 Let $T$ be a functor. A $T$-coalgebra is a pair $\langle A, \alpha\rangle$, where $A$ is a set and $\alpha$ is a function $\alpha: A \longrightarrow T A$. $A$ is called the carrier set or state set and $\alpha$ is called the transition structure.

Since $A=\operatorname{Dom} \alpha$ we often refer to $\langle A, \alpha\rangle$ simply as $\alpha$.

EXAMPLE 2.5 Some examples of coalgebras of polynomial functors:

- The three examples given above (Example 2.3) have coalgebras of the form $\langle X, \chi: X \longrightarrow T X\rangle$.

- The list $\langle 5,18,6,9\rangle$ can be represented by the $(T=\overline{1}+\overline{\mathbb{N}} \times \mathrm{Id})$-coalgebra 
$\langle X, \chi\rangle$, where $X=\left\{x_{0}, \ldots, x_{4}\right\}$ and

$$
\begin{aligned}
& \chi\left(x_{0}\right)=\left\langle 2,\left\langle 5, x_{1}\right\rangle\right\rangle \\
& \chi\left(x_{1}\right)=\left\langle 2,\left\langle 18, x_{2}\right\rangle\right\rangle \\
& \chi\left(x_{2}\right)=\left\langle 2,\left\langle 6, x_{3}\right\rangle\right\rangle \\
& \chi\left(x_{3}\right)=\left\langle 2,\left\langle 9, x_{4}\right\rangle\right\rangle \\
& \chi\left(x_{4}\right)=\langle 1, \star\rangle
\end{aligned}
$$

Recall that the top-level pair is from the disjoint union (coproduct).

- Consider the Mealy machine

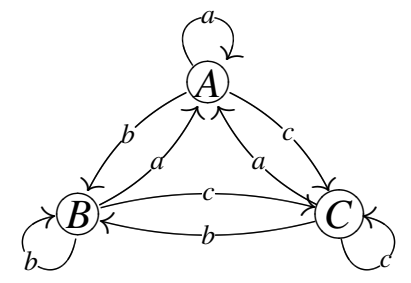

where each state outputs its label. It can be represented by the $(T=$ $\left.(\bar{O} \times \mathrm{Id})^{I}\right)$-coalgebra $\langle X, \chi\rangle$, where $I=\{a, b, c\}, O=\{A, B, C\}, X=\left\{x_{A}, x_{B}, x_{C}\right\}$ and

$$
\begin{aligned}
\chi\left(x_{A}\right)(a) & =\left\langle A, x_{A}\right\rangle \\
\chi\left(x_{A}\right)(b) & =\left\langle B, x_{B}\right\rangle \\
\chi\left(x_{A}\right)(c) & =\left\langle C, x_{C}\right\rangle \\
\chi\left(x_{B}\right)(a) & =\left\langle A, x_{A}\right\rangle \quad \ldots
\end{aligned}
$$

Definition 2.6 Let $\langle A, \alpha\rangle$ and $\langle B, \beta\rangle$ be $T$-coalgebras. A function $f: A \longrightarrow$ $B$ is a $(T-)$ morphism if $\beta \circ f=T f \circ \alpha$, i.e. the following diagram commutes:

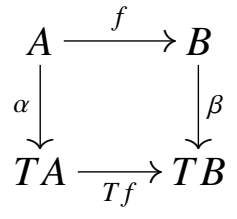


An isomorphism is a morphism which has an inverse that is also a morphism. The pair $\langle f, g\rangle$, of $T$-morphisms $f: A \longrightarrow B$ and $g: A \longrightarrow C$, is called a span.

EXAMPLE 2.7 Let $T=\overline{\mathbb{N}} \times \operatorname{Id}$ and $\langle A, \alpha\rangle$ and $\langle B, \beta\rangle$ be $T$-coalgebras (i.e. stream automata). We would intuitively say a function $f: A \longrightarrow B$ is a structure preserving transformation if $\operatorname{head}_{A}(a)=\operatorname{head}_{B}(f(a))$ and $f\left(\operatorname{tail}_{A}(a)\right)=$ tail $_{B}(f(a))$, and is hence a morphism as the following diagram commutes

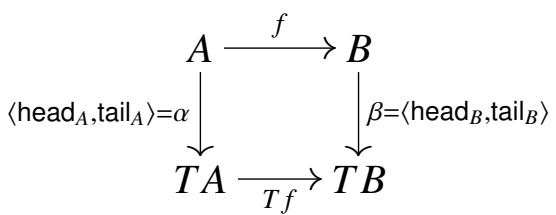

Suppose $\alpha$ represents the stream automaton for $\langle 1,2,3,4, \ldots\rangle$ and $\beta$ represents the stream automaton for $\langle 2,4,6,8, \ldots\rangle$, with states $a_{0}=\langle 1,2,3, \ldots\rangle$, $a_{1}=\langle 2,3,4, \ldots\rangle, b_{1}=\langle 4,6,8, \ldots\rangle$ and $b_{2}=\langle 6,8,10, \ldots\rangle$ etc. Then the function $f$ : map list $(\lambda x .2 x)$, that doubles every element, is a morphism from $\alpha$ to $\beta$ as, for example

$$
\begin{aligned}
& T f\left(\alpha\left(a_{0}\right)\right) \quad T f\left(\alpha\left(a_{5}\right)\right) \\
& =T f\left(\left\langle 1, a_{1}\right\rangle\right) \quad=T f\left(\left\langle 6, a_{6}\right\rangle\right) \\
& =\left\langle 2, b_{1}\right\rangle=\left\langle 12, b_{6}\right\rangle \\
& =\beta\left(b_{0}\right) \quad=\beta\left(b_{5}\right) \\
& =\beta\left(f\left(a_{0}\right)\right) \quad=\beta\left(f\left(a_{5}\right)\right)
\end{aligned}
$$

Proposition 2.8 Let $\langle A, \alpha\rangle,\langle B, \beta\rangle$ and $\langle C, \gamma\rangle$ be T-coalgebras and $f: A \longrightarrow B$ and $g: B \longrightarrow C$ be morphisms from $\alpha$ to $\beta$ and $\beta$ to $\gamma$ respectively.

(1) $\mathrm{id}_{A}$ is a morphism from $\alpha$ to $\alpha$.

(2) $g \circ f: A \longrightarrow C$ is a morphism from $\alpha$ to $\gamma$.

(3) if $f$ is a bijection then it is also an isomorphism.

\section{Proof}


(1) The diagram

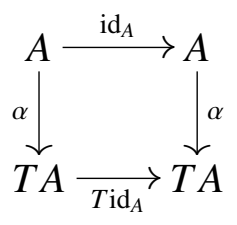

commutes as $T \mathrm{id}_{A}=\mathrm{id}_{T A}$ by definition (2.1).

(2) The diagram

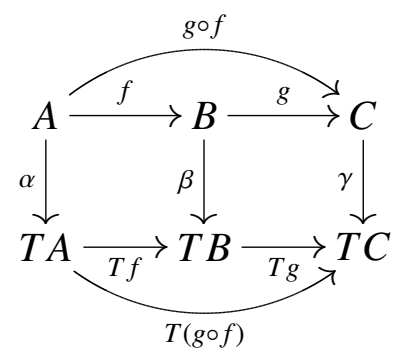

commutes as $T(g \circ f)=T g \circ T f$ by definition (2.1).

(3) Let $f$ be a bijection and $h: B \longrightarrow A$ be the inverse of $f$. Now

$$
\begin{aligned}
& \alpha \circ h & & \\
= & T h \circ T f \circ \alpha \circ h & & \text { as } T h \circ T f=\mathrm{id}_{T A} \\
= & T h \circ \beta \circ f \circ h & & \text { as } f \text { is a morphism } \\
= & T h \circ \beta & & \text { as } f \circ h=\mathrm{id}_{B}
\end{aligned}
$$

so $h$ is a morphism, and thus $f$ is an isomorphism.

Now we define pullbacks, which are useful notions we will use in proving results about bisimulations.

Definition 2.9 Let $A, B, C$ and $P$ be sets and $f: A \longrightarrow C, g: B \longrightarrow C$, $k: P \longrightarrow A$ and $l: P \longrightarrow B$ be functions with $f \circ k=g \circ l$. Then $\langle P, k, l\rangle$ is a (strong) pullback of $f$ and $g$ if, for any set $X$ and functions $i: X \longrightarrow A$ and $j: X \longrightarrow B$ with $f \circ i=g \circ j$, there exists a unique (called a mediating) 
function $h: X \longrightarrow P$ with $k \circ h=i$ and $l \circ h=j$ :

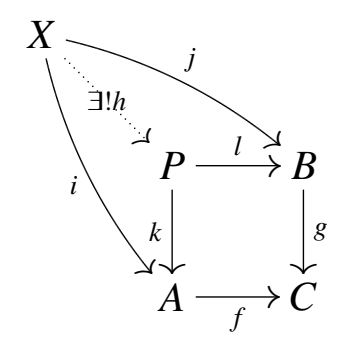

A weak pullback is defined as for a strong pullback, except that the mediating function need not be unique.

A functor $T$ is said to preserve pullbacks if it transforms pullbacks to pullbacks, i.e. if $\left\langle P, \pi_{1}, \pi_{2}\right\rangle$ is a pullback of $f$ and $g$ then $\left\langle T P, T \pi_{1}, T \pi_{2}\right\rangle$ is a pullback of $T f$ and $T g$. Similarly, $T$ preserves weak pullbacks if it transforms weak pullbacks to weak pullbacks.

This object from category theory is always defined for sets:

REMARK 2.10 The pullback of $f: A \longrightarrow C$ and $g: B \longrightarrow C$ is

$$
\left\langle P, \pi_{1}: P \longrightarrow A, \pi_{2}: P \longrightarrow B\right\rangle
$$

where

$$
P=\{\langle a, b\rangle \in A \times B \mid f(a)=g(b)\}
$$

THEOREM 2.11 Polynomial functors preserve both weak and strong pullbacks.

Proof By induction on the construction of the functor. Let $T$ be a polynomial functor and $\left\langle P, \rho_{1}, \rho_{2}\right\rangle$ be a pullback of $f: A \longrightarrow C$ and $g: B \longrightarrow C$.

CASE $T=$ Id: $T P=P$ is a pullback of $T f=f$ and $T g=g$.

CASE $T=\bar{D}: T P=D$ is obviously a pullback of $T f=\operatorname{id}_{D}$ and $T g=\mathrm{id}_{D}$ :

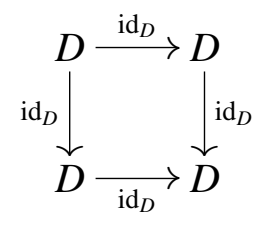


Now assume functors $T_{1}$ and $T_{2}$ preserve pullbacks.

CASE $T=T_{1}{ }^{D}$ : Suppose we have functions $k$ and $l$ from a set $X$ such that

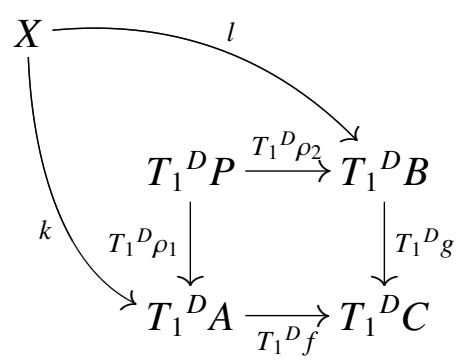

commutes. For every $x \in X$

$$
\begin{aligned}
& T_{1} f \circ(k(x)) \\
& =\left(T_{1}{ }^{D} f \circ k\right)(x) \quad \text { by definition } \\
& =\left(T_{1}{ }^{D} g \circ l\right)(x) \quad \text { as the diagram commutes } \\
& =T_{1} g \circ(l(x)) \quad \text { by definition }
\end{aligned}
$$

so we have

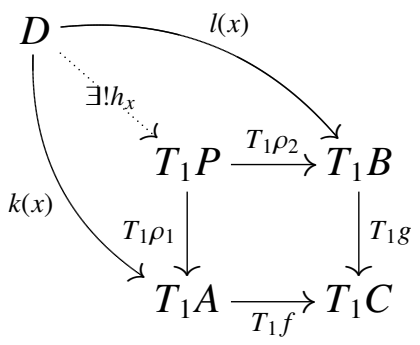

which is a pullback by the induction hypothesis with a unique $h_{x}$ : $D \longrightarrow T_{1} P$ as pictured. Define $h: X \longrightarrow T_{1}{ }^{D} P$ by $h(x)=h_{x}$. Now, for every $x \in X, T_{1}{ }^{D} \rho_{1} \circ h(x)=T_{1}{ }^{D} \rho_{1} \circ h_{x}=k(x)$ and $T_{1}{ }^{D} \rho_{2} \circ h(x)=$ $T_{1}{ }^{D} \rho_{2} \circ h_{x}=l(x)$ so

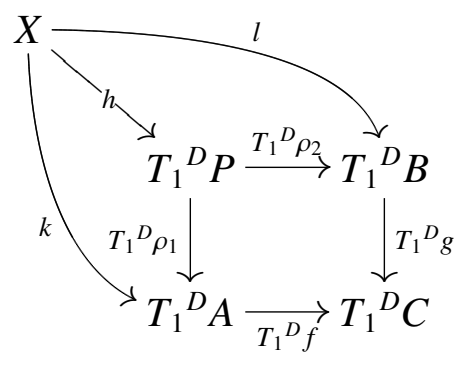


commutes. If there were some other $h^{\prime}: X \longrightarrow T_{1}{ }^{D} P$ with this property then it must disagree with $h$ for at least one $x \in X$. But this would violate the uniqueness of $h_{x}$. Hence $h$ is unique and $T_{1}{ }^{D} P$ is a pullback of $T_{1}{ }^{D} f$ and $T_{1}{ }^{D} g$.

CASE $T=T_{1} \times T_{2}$ : Suppose we have functions $k$ and $l$ from a set $X$ such that

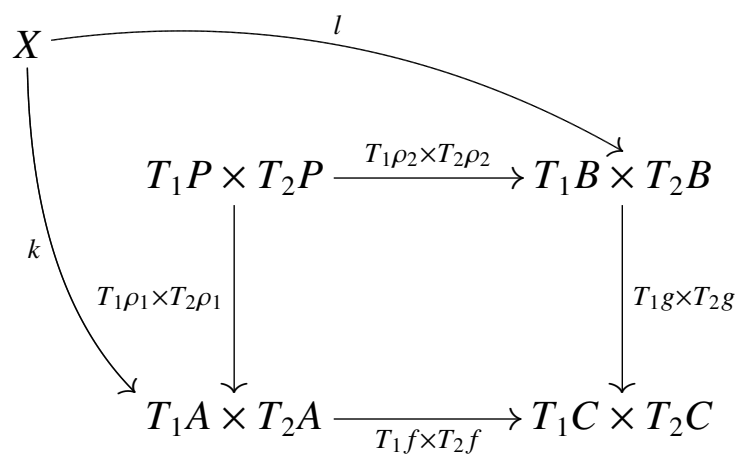

commutes. For each $j \in\{1,2\}, T_{j} P$ is a pullback with a unique $h_{j}$ :

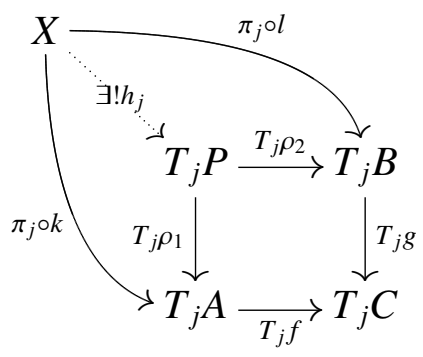

Define $h: X \longrightarrow T_{1} \times T_{2}: x \longmapsto\left\langle h_{1}(x), h_{2}(x)\right\rangle$. Now

$$
\begin{aligned}
& T_{1} \times T_{2} \rho_{1} \circ h(x) \\
& T_{1} \times T_{2} \rho_{2} \circ h(x) \\
& =\left\langle T_{1} \rho_{1} \circ h_{1}(x), T_{2} \rho_{1} \circ h_{2}(x)\right\rangle \\
& \text { and }=\left\langle T_{1} \rho_{2} \circ h_{1}(x), T_{2} \rho_{2} \circ h_{2}(x)\right\rangle \\
& =\left\langle\pi_{1} \circ k(x), \pi_{2} \circ k(x)\right\rangle \\
& =\left\langle\pi_{1} \circ l(x), \pi_{2} \circ l(x)\right\rangle \\
& =k(x) \\
& =l(x)
\end{aligned}
$$

and $h$ is unique due to the uniqueness of $h_{1}$ and $h_{2}$, so $T_{1} \times T_{2} P$ is a pullback of $T_{1} \times T_{2} f$ and $T_{1} \times T_{2} g$. 
CASE $T=T_{1}+T_{2}$ : Suppose we have functions $k$ and $l$ from a set $X$ such that

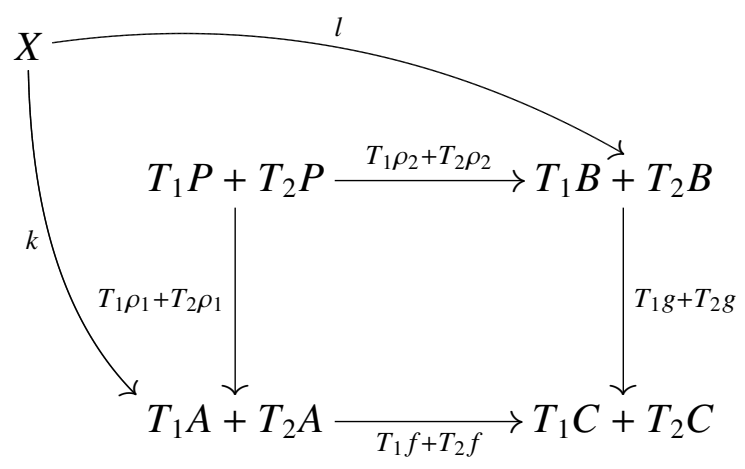

commutes. Then, for $j \in\{1,2\}, k(x) \in \operatorname{Dom} \varepsilon_{j}$ iff $l(x) \in \operatorname{Dom} \varepsilon_{j}$ :

$$
\begin{aligned}
& k(x) \in \operatorname{Dom} \varepsilon_{j} \\
& \text { iff }\left(T_{1} f+T_{2} f\right)(k(x)) \in \text { Dom } \varepsilon_{j} \text { by the definition of } T_{1} f+T_{2} f \\
& \text { iff }\left(T_{1} g+T_{2} g\right)(l(x)) \in \text { Dom } \varepsilon_{j} \text { as the diagram commutes } \\
& \text { iff } l(x) \in \operatorname{Dom} \varepsilon_{j} \quad \text { by the definition of } T_{1} g+T_{2} g
\end{aligned}
$$

Define $X_{j}=\left\{x \in X \mid k(x) \in\right.$ Dom $\left.\varepsilon_{j}\right\}$ for each $j \in\{1,2\}$. (Note that $X_{1} \cup$ $X_{2}=X$ and $X_{1} \cap X_{2}=\varnothing$.) Define $k_{1}: X_{1} \longrightarrow T_{1} A$ by $k_{1}(x)=\varepsilon_{1}(k(x))$ and similarly define $k_{2}: X_{2} \longrightarrow T_{2} B, l_{1}: X_{1} \longrightarrow T_{1} A$ and $l_{2}: X_{2} \longrightarrow T_{2} B$. For each $j \in\{1,2\}, T_{j} P$ is a pullback with a unique $h_{j}$ :

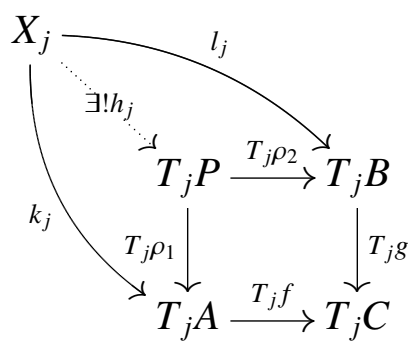

Now define $h: X \longrightarrow T_{1}+T_{2} P$ by $h(x)=\left\{\begin{array}{ll}\iota_{1}\left(h_{1}(x)\right) & \text { if } x \in X_{1} \\ \iota_{2}\left(h_{2}(x)\right) & \text { if } x \in X_{2}\end{array}\right.$. For each 


$$
\begin{aligned}
& j \in\{1,2\} \text { and } x \in X_{j} \\
& T_{1}+T_{2} \rho_{1} \circ h(x) \\
& =T_{1}+T_{2} \rho_{1} \circ \iota_{j} \circ h_{j}(x) \\
& =T_{j} \rho_{1} \circ h_{j}(x) \\
& =k_{j}(x) \\
& =k(x) \\
& \begin{aligned}
& T_{1}+T_{2} \rho_{2} \circ h(x) \\
= & T_{1}+T_{2} \rho_{2} \circ \iota_{j} \circ h_{j}(x) \\
\text { and } \quad & T_{j} \rho_{2} \circ h_{j}(x) \\
= & l_{j}(x) \\
= & l(x)
\end{aligned}
\end{aligned}
$$

$h$ is unique by the uniqueness of $h_{1}$ and $h_{2}$ so $T_{1}+T_{2} P$ is a pullback of $T_{1}+T_{2} f$ and $T_{1}+T_{2} g$.

Hence all polynomial functors preserve (strong) pullbacks. For weak pullbacks repeat the proof without the uniqueness properties.

\subsubsection{Bisimulation}

Definition 2.12 (ACZEL AND MENDleR [1]) For $T$-coalgebras $\langle A, \alpha\rangle$ and $\langle B, \beta\rangle$ a relation $R \subseteq A \times B$ is a (T-)bisimulation from $\alpha$ to $\beta$ if there exists a transition structure $\rho: R \longrightarrow T R$ on $R$ such that the projections are morphisms, i.e. the following diagram commutes:

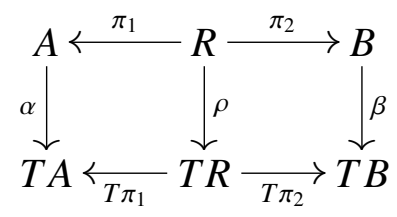

A bisimulation equivalence is a bisimulation that is also an equivalence relation. Two states $a \in A$ and $b \in B$ are bisimilar if there exists a bisimulation $R \subseteq A \times B$ with $\langle a, b\rangle \in R$.

EXAMPLE 2.13 Consider the systems below of the functor $T=\overline{1}+\operatorname{Id}^{I}$, where $I=\{c, d\}$, which only give output on termination after a (possibly infinite) sequence of input.
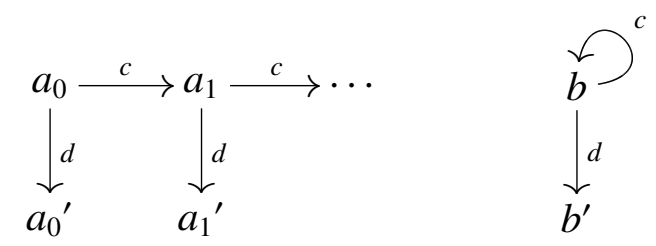
The system on the right is the coalgebra $\langle B, \beta\rangle$, where $B=\left\{b, b^{\prime}\right\}$ and $\beta(b)(c)=b, \beta(b)(d)=b^{\prime}$ and $\beta\left(b^{\prime}\right)=\star$. The system on the left is the coalgebra $\langle A, \alpha\rangle$, where $A=\left\{a_{0}, a_{0}{ }^{\prime}, a_{1}, a_{1}{ }^{\prime}, a_{2}, \ldots\right\}$ and, for all $i, \alpha\left(a_{i}\right)(c)=a_{i+1}$, $\alpha\left(a_{i}\right)(d)=a_{i}{ }^{\prime}$ and $\alpha\left(a_{i}{ }^{\prime}\right)=\star$.

The relation $R=\left\{\left\langle a_{i}, b\right\rangle\right\} \cup\left\{\left\langle a_{i}^{\prime}, b^{\prime}\right\rangle\right\}$ is a bisimulation from $\alpha$ to $\beta$ as the function $\rho: R \longrightarrow T R: r \longmapsto\left\langle\alpha\left(\pi_{1}(r)\right), \beta\left(\pi_{2}(r)\right)\right\rangle$ is a transition structure for $R$ and the projections $\pi_{1}$ and $\pi_{2}$ are obviously morphisms from $\rho$ to $\alpha$ and $\rho$ to $\beta$ respectively.

THEOREM 2.14 Let $\alpha$ and $\beta$ be T-coalgebras. A function $f: A \longrightarrow B$ is a morphism from $\alpha$ to $\beta$ iff its graph $G(f)=\{\langle a, f(a)\rangle \mid a \in A\}$ is a bisimulation from $\alpha$ to $\beta$.

Proof Consider the diagram

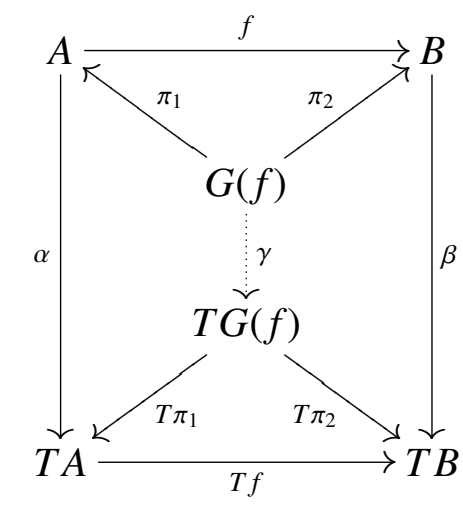

$(\Leftarrow)$ Let $G(f)$ be a bisimulation from $\alpha$ to $\beta$ and $\gamma: G(f) \longrightarrow T(G(f))$ be a transition structure for $G(f)$. The projections $\pi_{1}: G(f) \longrightarrow A$ and $\pi_{2}: G(f) \longrightarrow B$ are morphisms and so is $\pi_{1}^{-1}$, by Proposition 2.8(3), as $\pi_{1}$ is bijective. $f=\pi_{2} \circ \pi_{1}^{-1}$ thus, by Proposition 2.8(2), $f$ is a morphism.

$\Leftrightarrow$ Conversely, suppose $f$ is a morphism. Take $\left(T \pi_{1}\right)^{-1} \circ \alpha \circ \pi_{1}$ as a 
transition structure, $\gamma$, on $G(f) . \pi_{1}$ and $\pi_{2}$ are both morphisms:

$$
\begin{aligned}
& T \pi_{1} \circ \gamma \\
& =T \pi_{1} \circ\left(\left(T \pi_{1}\right)^{-1} \circ \alpha \circ \pi_{1}\right) \\
& =\alpha \circ \pi_{1} \\
& T \pi_{2} \circ \gamma \\
& =T \pi_{2} \circ\left(\left(T \pi_{1}\right)^{-1} \circ \alpha \circ \pi_{1}\right) \\
& =T\left(\pi_{2} \circ \pi_{1}^{-1}\right) \circ \alpha \circ \pi_{1} \\
& =T f \circ \alpha \circ \pi_{1} \\
& =\beta \circ f \circ \pi_{1} \\
& =\beta \circ \pi_{2}
\end{aligned}
$$

Thus morphisms are sometimes called functional bisimulations.

Bisimulations have a characterization in terms of liftings of relations, due to [15, 16], which we use later to define another characterization with path functions.

DEFINITION 2.15 For a relation $R \subseteq A \times B$ the lifting under a functor $T$ is a relation $R^{T} \subseteq T A \times T B$ defined inductively as:

$$
\begin{aligned}
R^{\bar{D}} & =\Delta_{D}=\{\langle d, d\rangle \mid d \in D\} \\
R^{\mathrm{Id}} & =R \\
R^{T_{1} \times T_{2}} & =\left\{\langle x, y\rangle \mid \pi_{1} x R^{T_{1}} \pi_{1} y \text { and } \pi_{2} x R^{T_{2}} \pi_{2} y\right\} \\
R^{T_{1}+T_{2}} & =\left\{\left\langle\iota_{1} x, \iota_{1} y\right\rangle \mid x R^{T_{1}} y\right\} \cup\left\{\left\langle\iota_{2} x, \iota_{2} y\right\rangle \mid x R^{T_{2}} y\right\} \\
R^{T_{1} D} & =\left\{\langle f, g\rangle \mid \forall d \in D, f(d) R^{T_{1}} g(d)\right\}
\end{aligned}
$$

THEOREM $2.16 R \subseteq A \times B$ is a bisimulation from $\alpha$ to $\beta$ iff for every $a \in A$ and $b \in B$

$$
a \text { R b implies } \alpha(a) R^{T} \beta(b)
$$

Now we prove some properties of bisimulations.

THEOREM 2.17 Let $f: A \longrightarrow C$ and $g: B \longrightarrow C$ be morphisms of $T$-coalgebras $\alpha$ to $\gamma$ and $\beta$ to $\gamma$, respectively. A weak pullback $\left\langle P \subseteq A \times B, \pi_{1}, \pi_{2}\right\rangle$ of $f$ and $g$ is a bisimulation from $\alpha$ to $\beta$. 
Proof Let $P \subseteq A \times B$ and

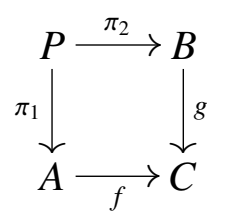

be a weak pullback. $T$ preserves weak pullbacks by Theorem 2.11, so

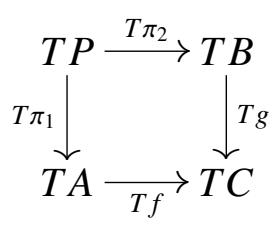

is also a weak pullback. But since

$$
\begin{aligned}
& T f \circ\left(\alpha \circ \pi_{1}\right) \\
& =\gamma \circ f \circ \pi_{1} \quad \text { as } f \text { is a morphism } \\
& =\gamma \circ g \circ \pi_{2} \quad \text { as } P \text { is a pullback } \\
& =T g \circ\left(\beta \circ \pi_{2}\right) \quad \text { as } g \text { is a morphism }
\end{aligned}
$$

there exists a function $\rho: P \longrightarrow T P$ with $\alpha \circ \pi_{1}=T \pi_{1} \circ \rho$ and $\beta \circ \pi_{2}=T \pi_{2} \circ \rho$ :

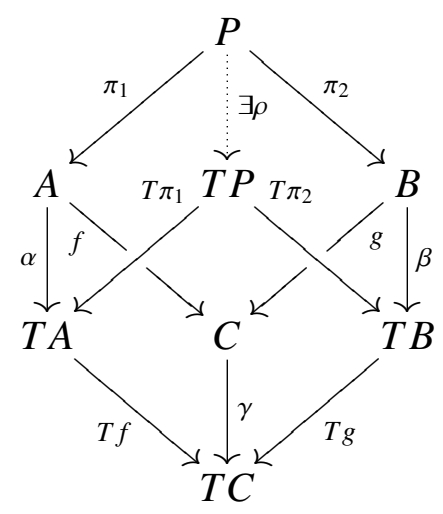

Thus $\langle P, \rho\rangle$ is a $T$-coalgebra and $\pi_{1}$ and $\pi_{2}$ are morphisms. Hence $P$ is a bisimulation from $\alpha$ to $\beta$.

Proposition 2.18 The diagonal $\Delta_{A}=\{\langle a, a\rangle \mid a \in A\}$ of a T-coalgebra $\langle A, \alpha\rangle$ is a bisimulation.

Proof Follows from Theorem 2.14 and the fact that $\Delta_{\alpha}$ is the graph of the identity $\mathrm{id}_{A}: A \longrightarrow A$. 
THEOREM 2.19 If $\langle R, \rho\rangle$ is a bisimulation from $\alpha$ to $\beta$ then so is its inverse $R^{-1}$. Proof Let $i$ be the isomorphism $R \longrightarrow R^{-1}:\langle a, b\rangle \longrightarrow\langle b, a\rangle$. Then

$$
\left\langle R^{-1}, T(i) \circ \rho \circ i^{-1}\right\rangle
$$

is a bisimulation from $\beta$ to $\alpha$ :

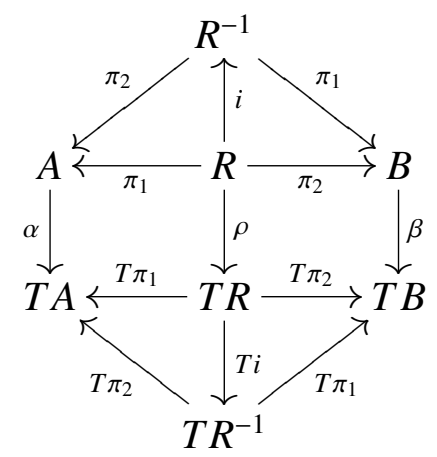

The next lemma says that the image of a span is a bisimulation, which we use to prove that the composition and union of bisimulations are bisimulations.

LEMMA 2.20 Let $f: A \longrightarrow B$ and $g: A \longrightarrow C$ be morphisms from $T$-coalgebras $\alpha$ to $\beta$ and $\alpha$ to $\gamma$ respectively. The image of the span of $f$ and $g,\langle f, g\rangle(A)=$ $\{\langle f(a), g(a)\rangle \mid a \in A\}$, is a bisimulation from $\beta$ to $\gamma$.

Proof Consider the following diagram:

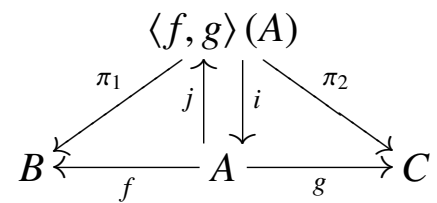

where the function $j$ is defined by $j(a)=\langle f(a), g(a)\rangle$ and the function $i$ is defined by $i(\langle f(a), g(a)\rangle)=a . j \circ i=\mathrm{id}_{\langle f, g\rangle(A)}$ and $\pi_{1}$ and $\pi_{2}$ are projections. Note that everything in the diagram commutes. The function $\rho=T j \circ \alpha \circ i$ is a transition structure for $\langle f, g\rangle(A)$. It follows that $\rho$ is a bisimulation from 
$\beta$ to $\gamma$ because

$$
\begin{aligned}
& T \pi_{1} \circ \rho \\
& \left.=T \pi_{1} \circ T\right) \circ \alpha \circ i \quad \text { by the definition of } \rho \\
& =T\left(\pi_{1} \circ j\right) \circ \alpha \circ i \quad \text { by the definition of functors } \\
& =T f \circ \alpha \circ i \quad \text { as the diagram commutes } \\
& =\beta \circ f \circ i \quad \text { as } f \text { is a functor } \\
& =\beta \circ \pi_{1} \quad \text { as the diagram commutes }
\end{aligned}
$$

and similarly for $\pi_{2}$.

THEOREM 2.21 The composition $R \circ S$ of $T$-bisimulations $R \subseteq A \times C$ and $S \subseteq$ $C \times B$ is a bisimulation from $\alpha$ to $\beta$.

Proof $R \circ S$ is equal to the image $\left\langle r_{1} \circ x_{1}, s_{2} \circ x_{2}\right\rangle(X)$ of the pullback:

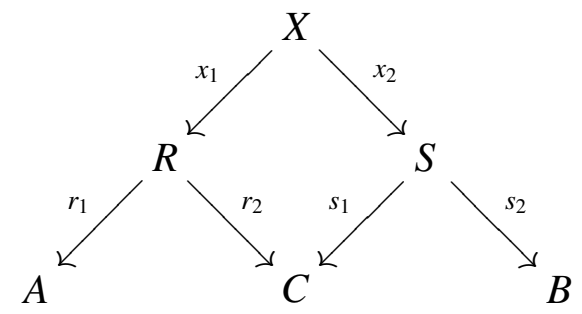

where $x_{i}, r_{i}$ and $s_{i}$ are projections. As $T$ preserves weak pullbacks, the pullback $X$ can be supplied with a transition structure, by Theorem 2.17 . such that the projections $x_{1}$ and $x_{2}$ are morphisms. Consequently both $r_{1} \circ x_{1}$ and $s_{2} \circ x_{2}$ are morphisms. By Lemma 2.20, $R \circ S$ is a bisimulation from $\alpha$ to $\beta$.

THEOREM 2.22 The union $\bigcup_{k} R_{k}$ of a family $\left\{R_{k}\right\}_{k}$ of bisimulations from coalgebras $\alpha$ to $\beta$ is again a bisimulation.

Proof $\bigcup_{k} R_{k}$ is the image of

$$
A \stackrel{k}{\longleftarrow} \sum_{i} R i \stackrel{l}{\longrightarrow} B
$$

where $k$ and $l$ are the componentwise projections. The coproduct of a family of coalgebras is a coalgebra, so it follows from Lemma 2.20 that the union is a bisimulation. 
COROLlary 2.23 The set of all bisimulations from $\alpha$ to $\beta$ is a complete lattice, with least upper bounds and greatest lower bounds given by

$$
\begin{gathered}
\bigvee_{k} R_{k}=\bigcup_{k} R_{k} \\
\bigwedge_{k} R_{k}=\bigcup\left\{R \mid R \text { is a bisimulation from } \alpha \text { to } \beta \text { with } R \subseteq \bigcap_{k} R_{k}\right\}
\end{gathered}
$$

In particular, the greatest bisimulation from $\alpha$ to $\beta$ exists, and is denoted by $\sim_{\langle\alpha, \beta\rangle}$. It is the union of all bisimulations:

$$
\sim_{\langle\alpha, \beta\rangle}=\bigcup\{R \mid R \text { is a bisimulation from } \alpha \text { to } \beta\}
$$

Consequently the greatest bisimulation on a coalgebra $\alpha$, written $\sim_{\alpha}$, is a bisimulation equivalence.

We write $\sim$ for the greatest bisimulation relation when the coalgebras are clear from context, and $\sim_{T}$ when explicit reference to the functor is needed.

\subsubsection{Final coalgebra}

DEFINITION 2.24 A $T$-coalgebra $\langle A, \alpha\rangle$ is final (or terminal] if for any $T$ coalgebra $\langle B, \beta\rangle$ there exists a unique morphism $!_{\beta}: B \longrightarrow A$

An important property of final coalgebras is that they are unique up to isomorphism.

Proposition 2.25 If $\langle A, \alpha\rangle$ and $\langle B, \beta\rangle$ are final $T$-coalgebras then there exists an isomorphism $f: A \longrightarrow B$.

Proof By finality there are unique morphisms $f: A \longrightarrow B$ and $g: B \longrightarrow$ $A$, which are mutual inverses and hence bijections. Thus, by Proposition 2.8(3), $f$ is an isomorphism.

\footnotetext{
1"We prefer final to terminal, which we associate with malady." [34 p. 41]
} 
THEOREM 2.26 (LAMBEK'S LEMMA [24]) If $\langle A, \alpha\rangle$ is a final T-coalgebra then $\alpha$ is an isomorphism.

Proof Since $\langle T A, T \alpha\rangle$ is a $T$-coalgebra there exists by the finality of $\alpha$ a unique morphism $f: T A \longrightarrow A$ :

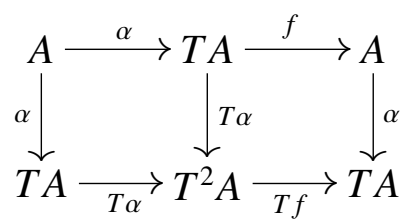

Again by the finality of $\alpha, f \circ \alpha=\mathrm{id}_{A}$. Now

$$
\begin{aligned}
& \alpha \circ f \\
& =T f \circ T \alpha \text { as } f \text { is a morphism } \\
& =T(f \circ \alpha) \quad \text { by the definition of functors } \\
& =T \operatorname{id}_{A} \quad \text { from above } \\
& =\operatorname{id}_{T A} \quad \text { by the definition of functors }
\end{aligned}
$$

Therefore $f$ is an inverse of $\alpha$, and $\alpha$ is an isomorphism.

THEOREM 2.27 (RUtTeN AND TURI [35]) Let $\langle B, \beta\rangle$ be a $T$-coalgebra, $\left\langle A_{T}, \alpha_{T}\right\rangle$ be a final T-coalgebra and $!_{\beta}: B \longrightarrow A_{T}$ the unique morphism from $\beta$ to $\alpha_{T}$. For every $b_{1}, b_{2} \in B, b_{1} \sim_{\beta} b_{2}$ iff $!_{\beta}\left(b_{1}\right)=!_{\beta}\left(b_{2}\right)$

Proof $\quad \Leftrightarrow$ Let $R$ be a bisimulation on $\beta$ with projections $\pi_{1}$ and $\pi_{2}$, and with $\left\langle b_{1}, b_{2}\right\rangle \in R$. The compositions $!_{\beta} \circ \pi_{1}$ and $!_{\beta} \circ \pi_{2}$ are morphisms from $R$ to $A_{T}$, by Proposition 2.8(2), and are equal by finality. In particular, $!_{\beta}\left(b_{1}\right)=$ $\left(!_{\beta} \circ \pi_{1}\right)\left\langle b_{1}, b_{2}\right\rangle=\left(!_{\beta} \circ \pi_{2}\right)\left\langle b_{1}, b_{2}\right\rangle=!_{\beta}\left(b_{2}\right)$.

$(\Leftarrow)$ Because $\Delta_{A_{T}}$ is a bisimulation on $A_{T}, !_{\beta}^{-1}\left(\Delta_{A_{T}}\right)$ is a bisimulation on $\beta$. If $!_{\beta}\left(b_{1}\right)=!_{\beta}\left(b_{2}\right)$ then $\left\langle b_{1}, b_{2}\right\rangle \in !_{\beta}^{-1}\left(\Delta_{A_{T}}\right)$, hence $b_{1} \sim_{\beta} b_{2}$

THEOREM 2.28 All polynomial functors have a final coalgebra.

This is a well-known result and there are many different methods for constructing a final coalgebra for an arbitrary polynomial functor. Rut- 
ten and Turi [35] use canonical solutions of domain equations; other authors [1, 2] exploit an anti foundation axiom in non-wellfounded set theory. In chapter 5 we shall give a model-theoretic construction similar to the canonical model construction in modal logic. This approach is also used by Kurz [23] and Rößiger [33], though for limited subsets of polynomial functors. Rößiger also constructs final coalgebras for all polynomial functors [33, §6] by using states that are sets, not of logical formulas, but of partial mappings from strings of 'positions' (c.f. basic paths, Definition 2.29) to observable output.

\subsection{Paths}

We use the notion of a path, from Jacobs [18] and Goldblatt [11], to make assertions about the structure and contents of transitions. A path specifies the location of one functor inside another and the induced path function allows us to label and make assertions about the result of a transition, which gives us another characterization of bisimulation.

DEFINITION 2.29 A path is a finite list of symbols of the kind $\pi_{j}, \varepsilon_{j}, e v_{d}$. We write $p . q$ for the concatenation of lists $p$ and $q$. We use the notation $T \stackrel{p}{\rightarrow} \rightarrow S$ to mean that $p$ is a path from functor $T$ to functor $S$, defined as:

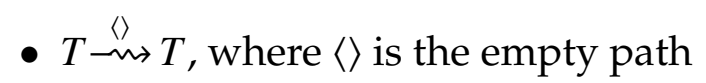

- $T_{1} \times T_{2} \stackrel{\pi_{j} \cdot q}{\leftrightarrow} S$, where $j \in\{1,2\}$ and $T_{j} \stackrel{q}{\stackrel{q}{\leftrightarrow}} S$

- $T_{1}+T_{2} \stackrel{\varepsilon_{j} \cdot q}{\leadsto} S$, where $j \in\{1,2\}$ and $T_{j} \stackrel{q}{\rightarrow} S$

- $T_{1} \stackrel{D^{e v_{d} \cdot q}}{\longrightarrow} S$ for every $d \in D$, where $T_{1} \stackrel{q}{\leftrightarrow} S$

A path $T \stackrel{p}{\rightarrow} S$ is a state path if $S=\mathrm{Id}$, an observation path if $S=\bar{D}$ and a basic path if it is either. 
REMARK 2.30 There is a path $T \rightarrow \leadsto$ iff $S$ is a component (is used in the construction) of $T$.

EXAMPLE 2.31 The following are examples of paths.

$$
\begin{aligned}
& \overline{1}+\mathrm{Id}^{\mathbb{N}} \stackrel{\varepsilon_{1}}{\leadsto} \overline{1} \quad \overline{\mathbb{N}} \times(\overline{1}+\mathrm{Id}) \stackrel{\pi_{1}}{\leadsto} \overline{\mathbb{N}} \\
& \overline{1}+\mathrm{Id}^{\mathbb{N}} \stackrel{\varepsilon_{2}}{\leadsto \mathrm{Id}^{\mathbb{N}}} \quad \overline{\mathbf{N}} \times(\overline{1}+\mathrm{Id}) \stackrel{\pi_{2}}{\leadsto} \overline{1}+\mathrm{Id} \\
& \overline{1}+\mathrm{Id}^{\mathbb{N}} \stackrel{\varepsilon_{2} \cdot e v_{1}}{\leadsto} \mathrm{Id} \quad \overline{\mathbb{N}} \times(\overline{1}+\mathrm{Id}) \stackrel{\pi_{2} \cdot \varepsilon_{1}}{\leadsto} \overline{1} \\
& \overline{1}+\mathrm{Id}^{\mathbb{N}} \stackrel{\varepsilon_{2} \cdot e_{2}+3}{\varkappa} \mathrm{Id} \quad \overline{\mathbb{N}} \times(\overline{1}+\mathrm{Id}) \stackrel{\pi_{2} \cdot \varepsilon_{2}}{\leadsto} \mathrm{Id}
\end{aligned}
$$

LEMMA 2.32 If $T$ contains no components of the form $S^{E}$ with $E$ uncountable, then the number of paths from $T$ is countable.

Proof Since $T$ is finitely constructed all paths are of length at most $n$, for some $n$. For each $0 \leqslant i \leqslant n$, let $X_{i}$ be the set of all paths of length $i$. This means that $X_{0}$ contains only the empty path \langle\rangle and is finite, and $\bigcup_{i \leqslant n} X_{i}$ contains all paths.

For each path $q \in X_{i+1}$ there is a path $p \in X_{i}$ such that

- $q=p . \pi_{j}, T \stackrel{p}{\leftrightarrow} S_{1} \times S_{2}$ and $j \in\{1,2\}$; or

- $q=p \cdot \varepsilon_{j}, T \stackrel{p}{\sim} S_{1}+S_{2}$ and $j \in\{1,2\}$; or

- $q=p \cdot e v_{e}, T \stackrel{p}{\sim} S^{E}$ and $e \in E$.

If all such sets $E$ are countable then $X_{i+1}$ can only be countably larger then $X_{i}$.

Thus $\bigcup_{i \leqslant n} X_{i}$ is countable, as it is the finite union of countable sets.

DEFINITION 2.33 A path $T \stackrel{p}{\leftrightarrow} S$ induces a partial function $p_{A}: T A \multimap S A$ for every set $A$ defined inductively as:

- \langle\rangle$_{A}: T A \longrightarrow T A$ is the identity function $\mathrm{id}_{T A}$ and is total.

- $\left(\pi_{j} \cdot p\right)_{A}=p_{A} \circ \pi_{j}$, the composition $T_{1} A \times T_{2} A \stackrel{\pi_{j}}{\longrightarrow} T_{j} A \stackrel{p_{A}}{\longrightarrow} S A$. Hence $x \in \operatorname{Dom}\left(\pi_{j} \cdot p\right)_{A}$ iff $\pi_{j}(x) \in \operatorname{Dom} p_{A}$. 
- $\left(\varepsilon_{j} \cdot p\right)_{A}=p_{A} \circ \varepsilon_{j}$, the composition $T_{1} A+T_{2} A \stackrel{\varepsilon_{j}}{\longrightarrow} T_{j} A \stackrel{p_{A}}{\longrightarrow} S A$. Hence $x \in \operatorname{Dom}\left(\varepsilon_{j} \cdot p\right)_{A}$ iff $x \in \operatorname{Dom} \varepsilon_{j}$ and $\varepsilon_{j}(x) \in \operatorname{Dom} p_{A}$.

- $\left(e v_{d} \cdot p\right)_{A}=p_{A} \circ e v_{d}$, the composition $(T A)^{D} \stackrel{e v_{d}}{\longrightarrow} T A \stackrel{p_{A}}{\longrightarrow} S A$. Hence $f \in \operatorname{Dom}\left(e v_{d} \cdot p\right)_{A}$ iff $f(d) \in \operatorname{Dom} p_{A}$.

EXAMPLE 2.34 Consider the $\mathbb{N}$ stream functor $T=\overline{\mathbb{N}} \times$ Id, with paths $T \stackrel{\pi_{1}}{\leadsto} \overline{\mathbb{N}}$ and $T \stackrel{\pi_{2}}{\leadsto}$ Id. For every $T$-coalgebra $\langle A, \alpha\rangle$ and state $a \in A$ the transition $\alpha(a)$ is a pair $\left\langle n, a^{\prime}\right\rangle$, where $n \in \mathbb{N}$ and $a^{\prime} \in A$. Thus the two path functions are

$$
\begin{aligned}
& \left(\pi_{1}\right)_{A}: T A \longmapsto \overline{\mathbb{N}} A=T A \longrightarrow \mathbb{N}:\left\langle n, a^{\prime}\right\rangle \longmapsto n \\
& \left(\pi_{2}\right)_{A}: T A \longrightarrow \operatorname{Id} A=T A \longrightarrow A:\left\langle n, a^{\prime}\right\rangle \longmapsto a^{\prime}
\end{aligned}
$$

Now consider the Mealy machine $\langle B, \beta\rangle$, a $\left((\overline{\mathbb{N}} \times \mathrm{Id})^{\mathbb{N}}\right)$-coalgebra that represents an automaton that cumulatively multiplies user input. At state $b_{n}$ the transition $\beta\left(b_{n}\right)$ gives a function $f_{n}: \mathbb{N} \longrightarrow \mathbb{N} \times B: m \longmapsto\left\langle n \times m, b_{n \times m}\right\rangle$. The path functions for this coalgebra are of the form

$$
\begin{aligned}
& \left(e v_{6}\right)_{B}\left(\beta\left(b_{7}\right)\right)=\left(e v_{6}\right)_{B}\left(f_{7}\right)=\left\langle 42, b_{42}\right\rangle \\
& \left(e v_{6} \cdot \pi_{1}\right)_{B}\left(\beta\left(b_{7}\right)\right)=42 \\
& \left(e v_{6} \cdot \pi_{2}\right)_{B}\left(\beta\left(b_{7}\right)\right)=b_{42} \\
& \left(e v_{29}\right)_{B}\left(\beta\left(b_{2}\right)\right)=\left(e v_{29}\right)_{B}\left(f_{2}\right)=\left\langle 58, b_{58}\right\rangle
\end{aligned}
$$

LEMMA 2.35 For every polynomial functor $T$, set $A$ and $x \in T A$ there exists $a$ basic path $T \stackrel{p}{\sim} \rightarrow S$ with $x \in \operatorname{Dom} p_{A}$.

Proof By induction on the construction of $T$.

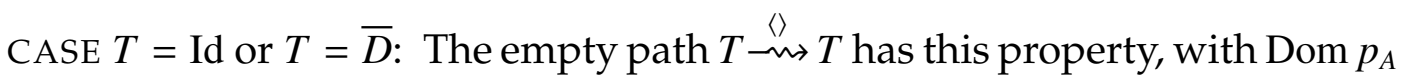
$=T A$.

Now assume the lemma holds for $T_{1}$ and $T_{2}$.

CASE $T=T_{1} \times T_{2}$ : If $x=\left\langle x_{1}, x_{2}\right\rangle \in T_{1} \times T_{2} A$ with $x_{1} \in T_{1} A$ and $x_{2} \in T_{2} A$ then, by the induction hypothesis, for both $j \in\{1,2\}$, there exists a basic 
path $T_{j} \stackrel{p_{j}}{\rightarrow} S_{j}$ with $x_{j} \in \operatorname{Dom}\left(p_{j}\right)_{A}$. Therefore $T_{1} \times T_{2} \stackrel{\pi_{j} \cdot p_{j}}{\rightarrow} S_{j}$ is a basic path with $x_{j} \in \operatorname{Dom}\left(\pi_{j} \cdot(p)_{j}\right)_{A}$.

CASE $T=T_{1}+T_{2}$ : If $x \in T_{1}+T_{2} A$ then $x=\iota_{j} T_{j} A$ and $\varepsilon_{j}(x) \in T_{j} A$. By the induction hypothesis there exists a basic path $T_{j} \stackrel{p}{\rightarrow} S$ with $\varepsilon_{j}(x) \in$ Dom $p_{A}$. So $T_{1}+T_{2} \stackrel{\varepsilon_{j} \cdot p}{\rightarrow} S$ is a basic path with $x \in \operatorname{Dom}\left(\varepsilon_{j} \cdot p\right)_{A}$.

CASE $T=T_{1}{ }^{D}$ : If $x \in T_{1}{ }^{D} A$ then, for all $d \in D, e v_{d}(x) \in T_{1} A$. By the induction hypothesis there exists a basic path $T_{1} \stackrel{p}{\rightarrow} S$ with $e v_{d}(x) \in \operatorname{Dom} p_{A}$. Then $T_{1}{ }^{D} \stackrel{e v_{d} \cdot p}{\rightarrow} S$ is a basic path with $x \in \operatorname{Dom}\left(e v_{d} \cdot p\right)_{A}$.

Now we combine the use of path functions with liftings of relations in order to obtain a characterization of bisimulation.

TheOREM 2.36 (Goldblatt [11]) For a polynomial functor $T$ let $R \subseteq A \times B$, $x \in T A$ and $y \in T B$. The following are equivalent:

(1) $x R^{T} y$

(2) $p_{A}(x) R^{S} p_{B}(y)$, for all paths $T \stackrel{p}{\sim} \rightarrow S$

(3) $p_{A}(x) R p_{B}(y)$, for all state paths $T \stackrel{p}{\sim} \rightarrow \mathrm{Id}$, and $p_{A}(x)=p_{B}(y)$, for all observation paths $T \stackrel{p}{\leftrightarrow} \rightarrow \bar{D}$

Proof $\quad(1) \Rightarrow(2)$ By induction on $T$.

CASE $T=\operatorname{Id}$ or $T=\bar{D}$ : The only path from $T$ is the empty path $T \stackrel{\langle\rangle}{\rightarrow} T$, with $p_{A}=\mathrm{id}_{T A}$ and $p_{B}=\mathrm{id}_{T B}$. So $x R^{T} y$ then $p_{A}(x) R^{T} p_{B}(y)$.

Now assume this holds for $T_{1}$ and $T_{2}$.

CASE $T=T_{1} \times T_{2}$ : If $x R^{T} y$ then $\pi_{1}(x) R^{T_{1}} \pi_{1}(y)$ and $\pi_{2}(x) R^{T_{2}} \pi_{2}(y)$. A path $T \stackrel{p}{\sim} \rightarrow S$ must have the form $T_{1} \times T_{2} \stackrel{\pi_{j} \cdot q}{\leadsto} S$, for $j \in\{1,2\}$, with $T_{j} \stackrel{q}{\rightarrow} \rightarrow S$. By the induction hypothesis $q_{A}\left(\pi_{j}(x)\right) R^{S} q_{B}\left(\pi_{j}(y)\right)$ so, since $p_{A}(x)=$ $q_{A}\left(\pi_{j}(x)\right)$ and $p_{B}(y)=q_{A}\left(\pi_{j}(y)\right)$, it follows that $p_{A}(x) R^{S} p_{B}(y)$. 
CASE $T=T_{1}+T_{2}$ : If $x R^{T} y$ then for some $j \in\{1,2\} x \in \iota_{j} T_{j} A, y \in \iota_{j} T_{j} B$ and $\varepsilon_{j}(x) R^{T_{j}} \varepsilon_{j}(y)$. A path $T \stackrel{p}{\sim} \rightarrow S$ must have the form $T_{1}+T_{2} \stackrel{e^{e k_{k} \cdot q} \rightarrow}{\rightarrow \text {, for }}$ some $k \in\{1,2\}$, with $T_{k} \stackrel{q}{\rightarrow} S$. If $k \neq j$ then neither $\varepsilon_{j}(x) \in \operatorname{Dom} q_{A}$ nor $\varepsilon_{j}(y) \in \operatorname{Dom} q_{B}$ so neither $p_{A}(x)$ nor $p_{B}(y)$ are defined.

If $k=j$ then by the induction hypothesis, since $\varepsilon_{j}(x) R^{T_{j}} \varepsilon_{j}(y)$, we have $q_{A}\left(\varepsilon_{j}(x)\right)$ defined iff $q_{B}\left(\varepsilon_{j}(y)\right)$ defined, and when both are defined $p_{A}(x)=q_{A}\left(\varepsilon_{j}(x)\right) R^{S} q_{B}\left(\varepsilon_{j}(y)\right)=p_{B}(y)$.

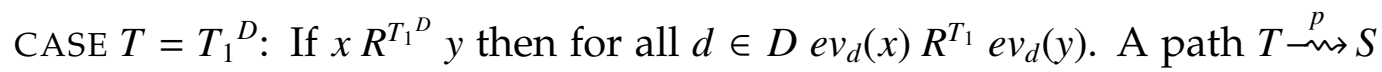
must have the form $T_{1} \stackrel{e v_{d} \cdot q}{\rightarrow} S$, for some $d \in D$, with $T_{1} \stackrel{q}{\rightarrow} S$. By the induction hypothesis $q_{A}\left(e v_{d}(x)\right) R^{S} q_{B}\left(e v_{d}(y)\right)$ so, since $p_{A}(x)=q_{A}\left(e v_{d}(x)\right)$ and $p_{B}(y)=q_{B}\left(e v_{d}(y)\right)$, it follows that $p_{A}(x) R^{S} p_{B}(y)$.

$(2) \Rightarrow(3)$ Immediate from definition.

(3) $\Rightarrow(1)$ By induction on $T$.

CASE $T=\operatorname{Id}$ or $T=\bar{D}$ : Let $p=\langle\rangle$. If (3) holds then we have $x=p_{A}(x) R^{T}$ $p_{B}(y)=y$, giving $(1)$.

Now assume this holds for $T_{1}$ and $T_{2}$.

CASE $T=T_{1} \times T_{2}$ : If $x \in T A$ and $y \in T B$ then, for each $j \in\{1,2\}, \pi_{j}(x) \in T_{j} A$ and $\pi_{j}(y) \in T_{j} B$. By Lemma 2.35 there exists a basic path $T_{j} \stackrel{q}{q} \rightarrow S$ with $\pi_{j}(x) \in \operatorname{Dom} q_{A}$ so, for $p=\pi_{j} . q, p_{A}(x)=\left(\pi_{j} . q\right)_{A}(x)=q_{A}\left(\pi_{j}(x)\right)$ is defined. If (3) holds for $T$, i.e. $p_{A}(x) R^{S} p_{B}(y)$, then $p_{B}(y)$ is defined and $\pi_{j}(y) \in \operatorname{Dom} q_{B}$.

In fact, for any basic path $T_{j}-q^{\prime} \leadsto S^{\prime}$, the above reasoning shows that $q_{A}^{\prime}\left(\pi_{j}(x)\right)$ is defined iff $q_{B}^{\prime}\left(\pi_{j}(y)\right)$ is defined, and furthermore that when they are both defined $\left(\pi_{j} \cdot q^{\prime}\right)_{A}(x) R^{S^{\prime}}\left(\pi_{j} \cdot q^{\prime}\right)_{B}(y)$ for $T$ ensures that $q_{A}^{\prime}(x) R^{S^{\prime}} q_{B}^{\prime}(y)$.

Thus (3) holds for $T_{j}$ so by induction hypothesis $\pi_{j}(x) R^{T_{j}} \pi_{j}(y)$. This is true for both $j \in\{1,2\}$ so we can conclude $x R^{T} y$. 
CASE $T=T_{1}+T_{2}$ : If $x \in T A$ and $y \in T B$ then, for exactly one $j \in\{1,2\}$, $x \in \iota_{j} T_{j} A$ and so $\varepsilon_{j}(x) \in T_{j} A$. By Lemma 2.35 there exists a basic path $T_{j} \stackrel{q}{\rightarrow} S$ with $\varepsilon_{j}(x) \in \operatorname{Dom} q_{A}$ so, for $p=\varepsilon_{j} \cdot q, p_{A}(x)=\varepsilon_{j} \cdot q_{A}(x)=$ $q_{A}\left(\varepsilon_{j}(x)\right)$ is defined. If (3) holds for $T$, i.e. $p_{A}(x) R^{S} p_{B}(y)$, then $p_{B}(y)=$ $\left(\varepsilon_{j} \cdot q\right)_{B}(y)$ is defined, so $y \in \iota_{j} T_{j} B$ and $\varepsilon_{j}(y) \in \operatorname{Dom} q_{B}$.

In fact, for any basic path $T_{j} \stackrel{q^{\prime}}{\rightarrow} S^{\prime}$, the above reasoning shows that $q_{A}^{\prime}\left(\varepsilon_{j}(x)\right)$ is defined iff $q_{B}^{\prime}\left(\varepsilon_{j}(y)\right)$ is defined, and when they are both defined $\left(\varepsilon_{j} \cdot q^{\prime}\right)_{A}(x) R^{S^{\prime}}\left(\varepsilon_{j} \cdot q^{\prime}\right)_{B}(y)$ for $T$ ensures $q_{A}^{\prime}\left(\varepsilon_{j}(x)\right) R^{S^{\prime}} q_{B}^{\prime}\left(\varepsilon_{j}(y)\right)$.

Thus (3) holds for $T_{j}$ so by induction hypothesis $\varepsilon_{j}(x) R^{T_{j}} \varepsilon_{j}(y)$, which implies $x R^{T} y$.

CASE $T=T_{1}{ }^{D}$ : If $x \in T A$ and $y \in T B$ then, for all $d \in D, e v_{d} \in T_{1} A$ and $e v_{d} \in T_{1} B$. By Lemma 2.35 there exists a basic path $T_{1} \stackrel{q}{\rightarrow} \rightarrow S$ with $e v_{d}(y) \in \operatorname{Dom} q_{B}$ so, for $p=e v_{d} \cdot q, p_{B}(y)=\left(e v_{d} \cdot q\right)_{B}(y)=q_{B}\left(e v_{d}(y)\right)$ is defined. If (3) holds for $T$, i.e. $p_{A}(x) R^{S} p_{B}(y)$, then $p_{A}(x)$ is defined and $e v_{d}(x) \in \operatorname{Dom} q_{A}$.

In fact, for any basic path $T_{1} \stackrel{q^{\prime}}{\rightarrow} S^{\prime}$, the above reasoning shows that $q_{B}^{\prime}\left(e v_{d}(y)\right)$ is defined iff $q_{A}^{\prime}\left(e v_{d}(x)\right)$, and when they are both defined (3) for $T$ ensures that $q_{A}^{\prime}\left(e v_{d}(x)\right) R^{S^{\prime}} q_{B}^{\prime}\left(e v_{d}(y)\right)$.

Thus (3) holds for $T_{1}$ so by induction hypothesis $e v_{d}(x) R^{T_{1}} e v_{d}(y)$. This is true for all $d \in D$ so we can conclude $x R^{T} y$.

Now we have the desired "dynamic" characterization of bisimulations and, as a consequence, morphisms.

THEOREM 2.37 (GoldBLATT [11]) Let $\langle A, \alpha\rangle$ and $\langle B, \beta\rangle$ be T-coalgebras. $R \subseteq$ $A \times B$ is a $T$-bisimulation iff $a R$ implies

$$
p_{A}(\alpha(a))=p_{B}(\beta(b))
$$

for every observation path $T \stackrel{p}{\rightarrow} \bar{D}$ and

$$
p_{A}(\alpha(a)) R p_{B}(\beta(b))
$$


for every state path $T \stackrel{p}{\rightarrow} \rightarrow \mathrm{Id}$.

Proof By Theorem 2.36 the latter half of the biconditional is equivalent to Theorem 2.16 ,

COROllary 2.38 (GoldBlatt [11]) Let $\langle A, \alpha\rangle$ and $\langle B, \beta\rangle$ be T-coalgebras. $f: A \longrightarrow B$ is a morphism from $\alpha$ to $\beta$ iff

$$
p_{A}(\alpha(a))=p_{B}(\beta(f(a)))
$$

for every observation path $p$ and

$$
f\left(q_{A}(\alpha(a))\right)=q_{B}(\beta(f(a)))
$$

for every state path $q$.

Proof By Theorem 2.14 $f$ is a morphism from $\alpha$ to $\beta$ iff its graph, $G(f)$, is a bisimulation from $\alpha$ to $\beta$. Now apply Theorem 2.37 to $G(f)$.

In the preceding theorems we saw that bisimulation is indeed a structure preserving relation. We will explicitly clarify this in a form that is needed in the next chapter.

COROLlary 2.39 If $R \subseteq A \times B$ is a T-bisimulation from $\alpha$ to $\beta$ and $a R$ then for all paths $T \stackrel{p}{\rightarrow} S$

$$
\alpha(a) \in \operatorname{Dom} p_{A} \quad \text { iff } \quad \beta(b) \in \operatorname{Dom} p_{B}
$$

Proof By Theorems 2.16 and 2.36, $a R b$ implies $p_{A}(\alpha(a)) R^{S} p_{B}(\beta(b))$ for all paths $T \stackrel{p}{\sim} S$, which has the implicit assumption that $\alpha(a) \in \operatorname{Dom} p_{A}$ iff $\beta(b) \in \operatorname{Dom} p_{B}$. 


\section{Chapter 3}

\section{Syntax and Semantics of Formulas}

We now define a Hennessy-Milner style modal logic for a fixed polynomial functor, $T$, which we use throughout. We place no restrictions on $T$ other than that it be non-trivial. The models of the logic are $T$-coalgebras, and it is expressive enough to distinguish states up to bisimilarity.

Definition 3.1 The set of well-formed formulas (wff) $\Phi_{T}$ consists of the following formulas:

- $\perp$

- $(p) \downarrow$, for every path $T \stackrel{p}{\rightarrow} S$

- $(p) c$, for every observation path $T \stackrel{p}{\sim} \underset{D}{D}$ and $c \in D$

- $\varphi \rightarrow \psi$, for every $\varphi, \psi \in \Phi_{T}$

- $[p] \varphi$, for every state path $T \stackrel{p}{\sim} \underset{\sim}{\operatorname{Id}}$ and $\varphi \in \Phi_{T}$

We also use the following useful abbreviations:

$$
\begin{aligned}
\neg \varphi & :=\varphi \rightarrow \perp \\
\top & :=\neg \perp \\
(p) \uparrow & :=\neg(p) \downarrow \\
\langle p\rangle \varphi & :=\neg[p] \neg \varphi
\end{aligned}
$$




$$
\begin{aligned}
\varphi \vee \psi & :=\neg \varphi \rightarrow \psi \\
\varphi \wedge \psi & :=\neg(\neg \varphi \vee \neg \psi) \\
\varphi \leftrightarrow \psi & :=(\varphi \rightarrow \psi) \wedge(\psi \rightarrow \varphi)
\end{aligned}
$$

LEMMA 3.2 If all constant sets used in the construction of $T$ are countable then $\Phi_{T}$ is countable.

Proof By induction on the construction of $\Phi_{T}$. Define $\mathbb{D}=\{D \mid T \stackrel{p}{\rightarrow} \bar{D}$ for some $p\}$ and $\mathbb{E}=\left\{E \mid T \stackrel{p}{\rightsquigarrow} S^{E}\right.$ for some $p$ and $\left.S\right\}$. Let every $C \in \mathbb{D} \cup \mathbb{E}$ be countable and fix an enumeration $c_{1}, c_{2}, c_{3}, \ldots$ of each $C$.

For $n \geqslant 0$, let $\Phi_{n}$ be a subset of $\Phi_{T}$ restricted such that $\varphi \in \Phi_{n}$ contains

- no paths (i.e. subformulas $(p) \downarrow,(p) d,[p] \psi)$ with a symbol $e v_{c_{i}}$ where $i>n$ and $c_{i} \in C \in \mathbb{E}$

- no subformulas of the form $(p) c_{j}$ where $j>n$ and $c_{j} \in C \in \mathbb{D}$

- at most $n$ arrows $(\rightarrow)$

- at most $n$ boxes ([p] $\psi$ subformulas)

It follows that

- $\Phi_{0}=\{\perp\}$

- $\Phi_{n} \subset \Phi_{n+1}$, for every $n \geqslant 0$

- each $\Phi_{n}$ is finite

- $\bigcup_{i \geqslant 0} \Phi_{i}=\Phi_{T}$

Therefore $\Phi_{T}$ is countable. 
DEFINITION 3.3 We define the truth relation $\vDash$ inductively as follows for all $T$-coalgebras $\langle A, \alpha\rangle$, with $x \in A$ :

- $\alpha, x \not \models \perp$

- $\alpha, x=(p) \downarrow \quad$ iff $\alpha(x) \in \operatorname{Dom} p_{A}$

- $\alpha, x=(p) c \quad$ iff $\quad \alpha, x=(p) \downarrow$ and $p_{A}(\alpha(x))=c$

- $\alpha, x=[p] \varphi \quad$ iff $\quad \alpha, x \not \models(p) \downarrow$, or $\alpha, x \models(p) \downarrow$ and $\alpha, p_{A}(\alpha(x)) \vDash \varphi$

- $\alpha, x \models \varphi \rightarrow \psi \quad$ iff $\quad \alpha, x=\varphi$ implies $\alpha, x=\psi$

We say that $\varphi$ is true at $x$ in $\alpha$, or $x$ satisfies $\varphi$, if $\alpha, x \vDash \varphi$, and that $\varphi$ is valid in $\alpha, \alpha=\varphi$, if it is true in all states in $A$. We call the set $\{\psi \mid \alpha, x \models \psi\}$ the truth set for $x$.

The following definitions are useful also:

$$
\begin{array}{ccl}
\alpha \vDash \varphi & \text { iff } & (\forall x \in A) \alpha, x \models \varphi \\
\alpha, x=\Gamma & \text { iff } & (\forall \psi \in \Gamma) \alpha, x=\psi \\
\Gamma \models^{\alpha} \varphi & \text { iff } & (\forall x \in A) \alpha, x \models \Gamma \text { implies } \alpha, x \models \varphi \\
\Gamma \models_{\bar{T}} \varphi & \text { iff } & (\forall\langle B, \beta\rangle) \Gamma \models^{\beta} \varphi
\end{array}
$$

REMARK $3.4 \alpha, x \not \models \varphi$ iff $\alpha, x \models \neg \varphi$

\section{Proof}

$$
\begin{array}{lll}
\alpha, x & =\neg \varphi & \\
\text { iff } \quad \alpha, x \vDash \varphi \rightarrow \perp & \text { by the definition of } \neg \\
\text { iff } \quad \alpha, x \vDash \varphi \text { implies } \alpha, x \models \perp & \text { by the semantics of } \rightarrow \\
\text { iff } \quad \alpha, x \neq \varphi & \text { as } \alpha, x \neq \perp
\end{array}
$$

Satisfaction of formulas is invariant under the action of morphisms. Since morphisms are functional bisimulations it follows that logical equivalence and bisimilarity coincide.

LEMMA 3.5 Let $\langle A, \alpha\rangle$ and $\langle B, \beta\rangle$ be T-coalgebras and $f: A \longrightarrow B$ be a morphism from $\alpha$ to $\beta$. Then $\alpha, a \vDash \varphi$ iff $\beta, f(a) \vDash \varphi$ for every $\varphi \in \Phi_{T}$.

Proof By induction on the construction of $\varphi$ : 
$\operatorname{CASE} \varphi=\perp: \alpha, a \not \neq \perp$ and $\beta, f(a) \not \neq \perp$

$\operatorname{CASE} \varphi=(p) \downarrow$ : The graph $G(f)$ is a bisimulation by Theorem 2.14, so $\alpha(a) \in \operatorname{Dom} p_{A}$ iff $\beta(f(a)) \in \operatorname{Dom} p_{B}$, for all paths $p$, by Corollary 2.39 . So $\alpha, a \vDash(p) \downarrow$ iff $\alpha(a) \in \operatorname{Dom} p_{A}$ iff $\beta(f(a)) \in \operatorname{Dom} p_{B}$ iff $\beta, f(a) \vDash(p) \downarrow$.

CASE $\varphi=(p) c$ :

$\begin{array}{ll}\alpha, a \vDash(p) c & \\ \text { iff } \quad \alpha, a \vDash(p) \downarrow \text { and } p_{A}(\alpha(a))=c & \text { by the semantics of }(p) c \\ \text { iff } \beta, f(a) \vDash(p) \downarrow \text { and } p_{B}(\beta(f(a)))=c & \text { by the previous CASE and } \\ & \text { Corollary 2.38 } \\ \text { iff } \beta, f(a) \vDash(p) c & \text { by the semantics of } \rightarrow\end{array}$

Now assume the lemma holds for $\theta, \psi$.

$\operatorname{CASE} \varphi=\theta \rightarrow \psi$ :

$\alpha, a \vDash \theta \rightarrow \psi$

iff $\alpha, a=\theta$ implies $\alpha, a \vDash \psi \quad$ by the semantics of $\rightarrow$

iff $\beta, f(a) \vDash \theta$ implies $\beta, f(a) \vDash \psi$ by the induction hypothesis

iff $\beta, f(a) \vDash \theta \rightarrow \psi \quad$ by the semantics of $\rightarrow$

$\operatorname{CASE} \varphi=[p] \psi$ :
$\alpha, a \vDash[p] \psi$
iff $\quad \alpha, a \vDash(p) \downarrow$ implies $\alpha, p_{A}(\alpha(a)) \vDash \psi \quad$ by semantics of $[p] \psi$
iff $\beta, f(a) \vDash(p) \downarrow$ implies $\beta, f\left(p_{A}(\alpha(a))\right) \vDash \psi \quad$ by induction hyp.
iff $\beta, f(a) \vDash(p) \downarrow$ implies $\beta, p_{B}(\beta(f(a))) \vDash \psi \quad$ by Corollary 2.38
iff $\beta, f(a) \vDash[p] \psi$
by semantics of $[p] \psi$ 
Now we are able to show that logical equivalence coincides with bisimilarity.

THEOREM 3.6 Let $\langle A, \alpha\rangle$ and $\langle B, \beta\rangle$ be $T$-coalgebras with $a \in A$ and $b \in B$. The following are equivalent:

(1) $a \sim b$

(2) $\alpha, a \vDash \varphi$ iff $\beta, b \vDash \varphi$ for every $\varphi \in \Phi_{T}$

Proof $\quad(1) \Rightarrow(2)$ Let $\langle R, \rho\rangle$ be a bisimulation from $\alpha$ to $\beta$ and $a R b$. The projections $\pi_{1}: R \longrightarrow A$ and $\pi_{2}: R \longrightarrow B$ are morphisms so, by Lemma 3.5 . $\alpha, a \vDash \varphi$ iff $\rho,\langle a, b\rangle \vDash \varphi$ iff $\beta, b \vDash \varphi$.

$(1) \Leftarrow(2)$ Let $R=\{\langle a, b\rangle \in A \times B \mid a$ and $b$ are logically equivalent $\}$. To show that $R$ is a bisimulation relation, by Theorem 2.37, we need to show that

(a) $p_{A}(\alpha(a))=p_{B}(\beta(b))$, for every observation path $\forall T \stackrel{p}{\sim} \rightarrow \bar{D}$

(b) $p_{A}(\alpha(a)) R p_{B}(\beta(b))$, for every state path $\forall T \stackrel{p}{\sim} \rightarrow \mathrm{Id}$

Firstly, for all basic paths, $\alpha(a) \in \operatorname{Dom} p_{A}$ iff $\beta(b) \in \operatorname{Dom} p_{B}$ by Corollary 2.39 For (a),

$$
\begin{aligned}
& p_{A}(\alpha(a))=d \\
& \text { iff } \quad \alpha, a \vDash(p) d \quad \text { by the semantics of }(p) d \\
& \text { iff } \quad \beta, b \vDash(p) d \quad \text { by the definition of } R \\
& \text { iff } p_{B}(\beta(b))=d \quad \text { by the semantics of }(p) d
\end{aligned}
$$

For (b), if $\alpha(a) \in \operatorname{Dom} p_{A}$ then

$$
\begin{aligned}
& \alpha, p_{A}(\alpha(a)) \vDash \varphi \\
& \text { iff } \quad \alpha, a \vDash[p] \varphi \quad \text { by the semantics of }[p] \varphi \\
& \text { iff } \quad \beta, b=[p] \varphi \quad \text { by the definition of } R \\
& \text { iff } \beta, p_{B}(\beta(b)) \vDash \varphi \quad \text { by the semantics of }[p] \varphi
\end{aligned}
$$




\section{Chapter 4}

\section{Axiomatic Construction}

In this section we give the axioms and relation that define the logic in a purely syntactic manner.

DEFINITION 4.1 If $\varphi$ is a wff and $p_{1}, \ldots, p_{n}$ are state paths then we say that the wff $\left[p_{1}\right] \ldots\left[p_{n}\right] \varphi$ is a generalization of $\varphi$.

DEFINITION 4.2 Let the set of axioms, $\Sigma_{T}$, consist of the following wffs and their generalizations.

1. all propositional tautologies

2. $(\langle\rangle) \downarrow$

For each path $T \stackrel{p}{\sim} \rightarrow S_{1} \times S_{2}$ :

3. $(p) \downarrow \rightarrow\left(\left(p . \pi_{1}\right) \downarrow \wedge\left(p . \pi_{2}\right) \downarrow\right)$

For each path $T \stackrel{p}{\sim} \rightarrow S_{1}+S_{2}$ :

4. $(p) \downarrow \rightarrow\left(\left(p . \varepsilon_{1}\right) \downarrow \leftrightarrow \neg\left(p . \varepsilon_{2}\right) \downarrow\right)$

For each path $T \stackrel{p}{\sim} \rightarrow S^{D}$ and all $d \in D$ :

5. $(p) \downarrow \rightarrow\left(p . e v_{d}\right) \downarrow$ 
For each observation path $T \stackrel{p}{\sim} \bar{D}$ and all $c, d \in D$ such that $c \neq d$ :

6. $(p) c \rightarrow \neg(p) d$

7. $(p) d \rightarrow(p) \downarrow$

For each state path $T \stackrel{p}{\sim}$ Id:

8. $\neg[p] \varphi \rightarrow[p] \neg \varphi$

9. $(p) \downarrow \rightarrow \neg[p] \perp$

10. $(p) \uparrow \rightarrow[p] \perp$

11. $[p](\varphi \rightarrow \psi) \rightarrow([p] \varphi \rightarrow[p] \psi)$

The axioms 9 and 10 characterize the relationship between formulas of the form $(p) \downarrow$ and $[p] \varphi$, namely that $\perp$ is true at all states accessible via $p$ iff $p$ is "undefined." However, to characterize the relationship between formulas of the form $(p) \downarrow$ and $(p) d$ we need axiom 7 and its potentially infinite converse $\neg(p) d_{1} \wedge \neg(p) d_{2} \wedge \cdots \wedge(p) d_{n} \wedge \ldots \rightarrow(p) \uparrow$ (for $T \stackrel{p}{\sim} \leadsto \bar{D}$ and all $\left.d_{i} \in D\right)$. We do not allow infinite formulas but would like to allow countably infinite constant sets so we use a relation to capture this property.

Definition 4.3 The richness relation $\mathcal{R}_{T} \subseteq 2^{\Phi_{T}} \times \Phi_{T}$ is the set of all pairs of the form

$$
\langle\{\neg(p) d \mid d \in D\},(p) \uparrow\rangle
$$

for all observation paths $T \stackrel{p}{\sim} \rightarrow \bar{D}$.

The relation $\mathcal{R}_{T}^{+}$is defined as the smallest extension of $\mathcal{R}_{T}$ such that, for all state paths $q$, if $\langle\Sigma, \varphi\rangle \in \mathbb{R}_{T}^{+}$then $\langle[q] \Sigma,[q] \varphi\rangle \in \mathcal{R}_{T}^{+}$, where $[q] \Sigma=$ $\{[q] \psi \mid \psi \in \Sigma\}$.

A set $\Gamma \subseteq \Phi_{T}$ is rich if it is closed under $\mathcal{R}_{T}^{+}$.

LEMMA 4.4 If $T$ does not contain any components of the form $S^{E}$ with $E$ uncountable, then $\mathcal{R}_{T}$ is countable. 
Proof $\mathcal{R}_{T}$ contains exactly one pair for each observation path from $T$. If $T$ has no power components with uncountable constant sets then it has at most countably many paths, by Lemma 2.32 . If there are countably many paths then there are countably many observation paths, so $\mathcal{R}_{T}$ is countable too.

THEOREM 4.5 All axioms are valid in all $T$-coalgebras and $\Sigma \mathcal{R}_{T}^{+} \varphi$ implies $\left.\Sigma\right|_{T} \varphi$.

\section{Proof}

1. Propositional tautologies are valid from the semantics of $\perp$ and $\rightarrow$.

2. \langle\rangle$_{A}$ is always defined, so $\alpha, x \models(\langle\rangle) \downarrow$.

$3-5$. These three axioms follow from the definition of $p_{A}$ :

$\chi \in \operatorname{Dom} p \cdot \pi_{j_{A}}$ iff $\chi \in \operatorname{Dom} p_{A}$

$\chi \in \operatorname{Dom} p . e v_{d_{A}}$ iff $\chi \in \operatorname{Dom} p_{A}$

$\chi \in \operatorname{Dom} p \cdot \varepsilon_{j_{A}}$ iff $\chi \in \operatorname{Dom} p_{A}$ and $p_{A}(\chi) \in \operatorname{Dom} \varepsilon_{j}$

6. $\alpha, x \vDash(p) c \rightarrow \neg(p) d$ :

If $\alpha, x \models(p) c$ then $p_{A}(\alpha(x))=c$, so $p_{A}(\alpha(x)) \neq d$. Hence $\alpha, x \not \models(p) d$.

7. $\alpha, x \vDash(p) d \rightarrow(p) \downarrow$ :

$\alpha, x \vDash(p) d$ implies $\alpha, x \vDash(p) \downarrow$ by definition.

8. $\alpha, x \vDash\langle p\rangle \varphi \rightarrow[p] \varphi$ :

If $\alpha, x \vDash\langle p\rangle \varphi$ then $\alpha, x \vDash \neg[p] \neg \varphi$ and $\alpha, x \notin[p] \neg \varphi$ by definition. Therefore $\alpha, x \vDash(p) \downarrow$ and $\alpha, p_{A}(\alpha(x)) \vDash \neg \varphi$. Hence, $\alpha, p_{A}(\alpha(x)) \vDash \varphi$ and $\alpha, x \vDash[p] \varphi$.

9. $\alpha, x \models(p) \downarrow \rightarrow\langle p\rangle\rceil$ :

If $\alpha, x \vDash(p) \downarrow$ then $\alpha(x) \in \operatorname{Dom} p_{A}$. Therefore $\alpha, p_{A}(\alpha(x)) \not \models \perp$ and $\alpha, x \not \models[p] \perp$. From the definitions it follows that $\alpha, x \models\langle p\rangle \mathrm{T}$. 
10. $\alpha, x \vDash \neg(p) \downarrow \rightarrow[p] \varphi:$

If $\alpha, x \models \neg(p) \downarrow$ then $\alpha, x \not \models(p) \downarrow$ so $\alpha, x \not \models[p] \varphi$.

11. $\alpha, x \vDash[p](\varphi \rightarrow \psi) \rightarrow([p] \varphi \rightarrow[p] \psi)$ :

If $\alpha, x \vDash(p) \uparrow$ then $\alpha, x \vDash[p](\varphi \rightarrow \psi)$ and $\alpha, x \vDash[p] \varphi$ and $\alpha, x \vDash[p] \psi$ so let's assume $\alpha, x \vDash(p) \downarrow$. If $\alpha, x \vDash[p](\varphi \rightarrow \psi)$ then $\alpha, p_{A}(\alpha(x)) \vDash \varphi \rightarrow \psi$. Therefore $\alpha, p_{A}(\alpha(x)) \vDash \varphi$ implies $\alpha, p_{A}(\alpha(x)) \vDash \psi$ and $\alpha, x \vDash[p] \varphi$ implies $\alpha, x \vDash[p] \psi$.

Generalizations: inductively, let $\varphi$ be an axiom, or a generalization of an axiom, and $p$ a state path. If $\alpha, x \vDash(p) \uparrow$ then $\alpha, x \vDash[p] \varphi$ trivially. Otherwise it has been shown that $\alpha, p_{A}(\alpha(x)) \vDash \varphi$ so $\alpha, x \vDash[p] \varphi$ by definition.

Richness:

- Suppose $\langle\{\neg(p) d \mid d \in D\},(p) \uparrow\rangle \in \mathcal{R}_{T}$ and, for all $d \in D, \alpha, x=\neg(p) d$. Then $p_{A}(\alpha(x)) \neq d$ for each $d$, i.e. $\alpha(x) \notin \operatorname{Dom} p_{A}$, so $\alpha, x \not \neq(p) \downarrow$ and $\alpha, x \vDash(p) \uparrow$ by Remark 3.4 . Hence $\Sigma \mathcal{R}_{T} \varphi$ implies $\Sigma \models_{\bar{T}} \varphi$.

- Suppose $\Gamma \models_{\bar{T}} \varphi$. Then for some state path $q, T$-coalgebra $\alpha$ and state $x \in \operatorname{Dom} \alpha, \alpha, x \not \models(q) \downarrow$ implies $\alpha, x \vDash[q] \varphi$, and if $\alpha, x \models(q) \downarrow$

$\begin{array}{ccl} & \alpha,\left.x\right|_{\bar{T}}[q] \Gamma & \\ \text { implies } & \alpha,\left.x\right|_{\bar{T}}[q] \psi & \text { for each } \psi \in \Gamma \\ \text { implies } & \alpha,\left.q_{A}(\alpha(x))\right|_{\bar{T}} \psi & \text { by the semantics of }[q] \psi \\ \text { implies } & \alpha,\left.q_{A}(\alpha(x))\right|_{\overline{\bar{T}}} \Gamma & \\ \text { implies } & \alpha,\left.q_{A}(\alpha(x))\right|_{\bar{T}} \varphi & \text { as }\left.\Gamma\right|_{\bar{T}} \varphi \\ \text { implies } & \alpha,\left.x\right|_{\bar{T}}[q] \varphi & \text { by the semantics of }[q] \varphi\end{array}$

Thus $\mathcal{R}_{T} \subseteq F_{\bar{T}}$ and $\langle\Sigma, \varphi\rangle \in F_{\bar{T}}$ implies $\langle[p] \Sigma,[p] \varphi\rangle \in F_{\bar{T}} . \mathcal{R}_{T}^{+}$is the smallest relation satisfying these properties, hence $\Sigma \mathcal{R}_{T}^{+} \varphi$ implies $\Sigma \models_{T} \varphi$. 


\section{Chapter 5}

\section{Canonical coalgebras}

Here we define the canonical $T$-coalgebra, whose states consist of "maximal theories" of wffs. The construction is similar as for a Henkin proof of completeness in modal logic, as described in [7, 8], and this turns out to be a natural construction of a final $T$-coalgebra.

DEFINITION 5.1 For $R \subseteq 2^{\Phi_{T}} \times \Phi_{T}, \Delta \subseteq \Phi_{T}$ is a $T R$-theory if

- it contains the set $\Sigma_{T}$ of axioms

- it is closed under Detachment, i.e. $\varphi, \varphi \rightarrow \psi \in \Delta$ implies $\psi \in \Delta$

- it is closed under $R$, i.e. $\langle\Gamma, \varphi\rangle \in R$ and $\Gamma \subseteq \Delta$ implies $\varphi \in \Delta$.

DEFINITION 5.2 A set of wff, $\Gamma$, is negation complete if either $\varphi \in \Gamma$ or $\neg \varphi \in \Gamma$ for every $\varphi \in \Phi_{T} . \Gamma$ is a maximally $\perp$-free $T \mathcal{R}_{T}^{+}$-theory if it is a negation complete $T \mathcal{R}_{T}^{+}$-theory and $\perp \notin \Gamma$.

The use of the word 'maximal' in the previous definition is justified by the following lemma.

LeMmA 5.3 Let $\Gamma$ and $\Delta$ be $T R_{T}^{+}$-theories not containing $\perp$. If $\Gamma$ is negation complete and $\Gamma \subseteq \Delta$ then $\Gamma=\Delta$. 
Proof Let $\Gamma$ and $\Delta$ be $T R_{T}^{+}$-theories, $\Gamma$ be negation complete, $\perp \notin \Gamma, \perp \notin \Delta$ and $\Gamma \subseteq \Delta$.

Assume there is a formula $\varphi \in \Delta$ and $\varphi \notin \Gamma$. By negation completeness $\neg \varphi \in \Gamma$ so $\neg \varphi \in \Delta$. The tautology $\varphi \rightarrow(\neg \varphi \rightarrow \perp) \in \Delta$, so by Detachment $\perp \in \Delta$ (a contradiction). Hence $\Gamma=\Delta$.

LemmA 5.4 Let $\langle A, \alpha\rangle$ be a T-coalgebra. The truth set $\{\varphi \mid \alpha, x \vDash \varphi\}$ for $x \in A$ is a maximally $\perp$-free $T \mathcal{R}_{T}^{+}$-theory.

Proof $\{\varphi \mid \alpha, x \vDash \varphi\}$ is a $T R_{T}^{+}$-theory as it contains the axioms and is rich by Theorem 4.5 and is closed under detachment by the semantics of $\rightarrow$.

It is negation complete by Remark 3.4 , and it is $\perp$-free by the semantics of $\perp$.

The following two lemmas are important properties of maximally $\perp$ free $T R_{T}^{+}$-theories that are crucial in the construction of the canonical $T$ coalgebra.

LEMMA 5.5 Let $\Gamma$ be a maximally $\perp$-free $T R_{T}^{+}$-theory. For each observation path $T \stackrel{p}{\sim} \bar{D}$ if $(p) \downarrow \in \Gamma$ then $(p) d \in \Gamma$ for a unique $d \in D$.

Proof If $(p) \downarrow \in \Gamma$ then $(p) \uparrow \notin \Gamma$, as $\Gamma$ is closed under Detachment and contains the tautology $(p) \downarrow \rightarrow((p) \uparrow \rightarrow \perp)$ but not $\perp$. $\Gamma$ is also rich, so is closed under the pair $\langle\{\neg(p) d \mid d \in D\},(p) \uparrow\rangle \in \mathcal{R}_{T}^{+}$. But $(p) \uparrow \notin \Gamma$ so $\neg(p) d \notin \Gamma$, for at least one $d$, and $(p) d \in \Gamma$ by negation completeness. In fact $d$ is unique, as axiom 6, $(p) d \rightarrow \neg(p) d^{\prime}$, and Detachment ensure that $\neg(p) d^{\prime} \in \Gamma$ for every $d^{\prime} \in D$ where $d^{\prime} \neq d$.

LEMMA 5.6 For all state paths $p$, if $\Delta$ is a $T R_{T}^{+}$-theory and $(p) \downarrow \in \Delta$ then $\Delta_{p}=$ $\{\varphi \mid[p] \varphi \in \Delta\}$ is also a $T R_{T}^{+}$-theory. Furthermore, if $\Delta$ is a maximally $\perp$-free $T R_{T}^{+}-$ theory then so is $\Delta_{p}$.

Proof Let $\Delta$ be a $T \mathcal{R}_{T}^{+}$-theory and $(p) \downarrow \in \Delta$.

If $\perp \in \Delta$ then $\Delta=\Phi_{T}$ by the tautology $\perp \rightarrow \varphi \in \Delta$ and Detachment. Therefore $\Delta_{p}=\Delta$ and is a $T \mathcal{R}_{T}^{+}$-theory. Neither $\Delta$ nor $\Delta_{p}$ are maximally $\perp$-free $T R_{T}^{+}$-theories. 
Suppose $\perp \notin \Delta . \Delta_{p}$ contains all axioms by the generalization property and is closed under Detachment by axiom 11 . The $[p]$ closure property that extends $\mathcal{R}_{T}$ to $\mathcal{R}_{T}^{+}$ensures that $\Delta_{p}$ is also rich. Hence it too is a $T R_{T}^{+}$-theory.

Suppose $\Delta$ is negation complete also. Therefore for every $\varphi$ either $[p] \varphi \in$ $\Delta$ or $\neg[p] \varphi \in \Delta$. But $\neg[p] \varphi \rightarrow[p] \neg \varphi \in \Delta$, by axiom 8 , so either $[p] \varphi \in \Delta$ or $[p] \neg \varphi \in \Delta$, by Detachment. Thus either $\varphi \in \Delta_{p}$ or $\neg \varphi \in \Delta_{p}$, so $\Delta_{p}$ is negation complete.

From axiom $9(p) \downarrow \rightarrow \neg[p] \perp \in x$, so $\neg[p] \perp \in x$ by Detachment. If $\perp \in \Delta_{p}$ then $[p] \perp \in \Delta$, so $[p] \perp,[p] \perp \rightarrow \perp \in \Delta$, yielding $\perp \in \Delta$, by Detachment, which is a contradiction. Hence $\perp \notin \Delta_{p}$.

Now we can construct the canonical $T$-coalgebra $\left(A_{T}, \alpha_{T}\right)$, with the state set consisting of maximally $\perp$-free $T R_{T}^{+}$-theories and the transition structure defined inductively from the basic paths up.

DEFINITION $5.7 A_{T}=\left\{x \mid x\right.$ is a maximally $\perp$-free $T R_{T}^{+}$-theory $\}$

LEMMA 5.8 For every $T \stackrel{p}{\rightarrow} S$ there exists a partial function $\alpha_{p}: A_{T} \longleftrightarrow S A_{T}$ with Dom $\alpha_{p}=\{x \mid(p) \downarrow \in x\}$ such that, for every $S \stackrel{q}{\rightarrow} U$,

$$
\operatorname{Dom} \alpha_{p . q}=\left\{x \in A_{T} \mid x \in \operatorname{Dom} \alpha_{p} \text { and } \alpha_{p}(x) \in \operatorname{Dom} q_{A_{T}}\right\}
$$

and $\alpha_{p . q}=q_{A_{T}} \circ \alpha_{p}$ :

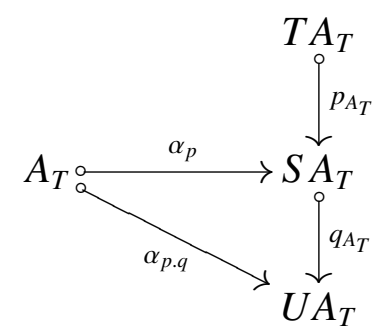

Proof We define $\alpha_{p}$ by induction on $S$.

CASE $T \stackrel{p}{\rightarrow} \rightarrow \bar{D}$ : By Lemma 5.5 there is a unique $(p) d \in x$. Let $\alpha_{p}(x)=d$.

CASE $T \stackrel{p}{\leadsto}$ Id: Let $\alpha_{p}(x)=x_{p}$ where $x_{p}=\{\varphi \mid[p] \varphi \in x\}$ and $x_{p} \in A_{T}$ by Lemma 5.6 . 
CASE $T \stackrel{p}{\sim} \rightarrow S_{1} \times S_{2}$ : Let $\alpha_{p}(x)=\left\langle\alpha_{p \cdot \pi_{1}}(x), \alpha_{p \cdot \pi_{2}}(x)\right\rangle$.

CASE $T \stackrel{p}{\rightarrow} S_{1}+S_{2}$ : If $\left(p \cdot \varepsilon_{j}\right) \downarrow \in x$ then let $\alpha_{p}(x)=\iota_{j} \alpha_{p . \varepsilon_{j}}(x)$. This is the case for exactly one $j \in\{1,2\}$.

CASE $T \stackrel{p}{\sim} S^{D}$ : for all $d \in D$, let $\alpha_{p}(x)(d)=\alpha_{p \cdot v_{d}}(x)$.

We prove the second part by induction on $S$ again.

CASE $S=\operatorname{Id}$ or $\bar{D}, q=\langle\rangle:$ In this case $p . q=p$ and $q_{A_{T}}=\operatorname{id}_{S A_{T}}$, so $\alpha_{p . q}=\alpha_{p}=$ $\operatorname{id}_{S A_{T}} \circ \alpha_{p}=q_{A_{T}} \circ \alpha_{p}$

Now assume that $\alpha_{s . r}=r_{A_{T}} \circ \alpha_{s}$ where $s=p . \sigma$ and $q=\sigma . r$ for some symbol $\sigma$.

CASE $S=S_{1} \times S_{2}, q=\pi_{j} . r$ : From the induction hypothesis we assume Dom $\alpha_{p \cdot \pi_{j}, r}=\operatorname{Dom} r_{A_{T}} \circ \alpha_{p \cdot \pi_{j}}$ and $\alpha_{p \cdot \pi_{j}, r}=r_{A_{T}} \circ \alpha_{p \cdot \pi_{j}}$. We know that $\pi_{j}$ is total and $(p) \downarrow \in x$ implies $\left(p . \pi_{j}\right) \downarrow \in x$ so $\operatorname{Dom} \alpha_{p . \pi_{j}}=\operatorname{Dom}\left(\pi_{j}\right)_{A_{T}} \circ \alpha_{p}$. From the definition of $\alpha_{p}$ we see $\alpha_{p \cdot \pi_{j}}=\pi_{j} \circ \alpha_{p}$. Hence, since $\left(\pi_{j} . r\right)_{A_{T}}=r_{A_{T}} \circ \pi_{j}$, $\operatorname{Dom} \alpha_{p, \pi_{j} . r}=\operatorname{Dom} \pi_{j} \cdot r_{A_{T}} \circ \alpha_{p}$ and $\alpha_{p, \pi_{j} . r}=\pi_{j} . r_{A_{T}} \circ \alpha_{p}$.

CASE $S=S_{1}{ }^{D}, q=e v_{d} . r$ : From the induction hypothesis we assume for every $d \in D \operatorname{Dom} \alpha_{p . e v_{d}, r}=\operatorname{Dom} r_{A_{T}} \circ \alpha_{p . e v_{d}}$ and $\alpha_{p . e v_{d} . r}=r_{A_{T}} \circ \alpha_{p . e v_{d}}$. We know $e v_{d}$ is total and $(p) \downarrow \in x$ implies $\left(p . e v_{d}\right) \downarrow \in x$, so $\operatorname{Dom} \alpha_{p . e v_{d}}=$ $\operatorname{Dom}\left(e v_{d}\right)_{A_{T}} \circ \alpha_{p}$. From the definition of $\alpha_{p}$ we see $\alpha_{p . e v_{d}}=e v_{d} \circ \alpha_{p}$. Hence, since $\left(e v_{d} \cdot r\right)_{A_{T}}=r_{A_{T}} \circ e v_{d}$, Dom $\alpha_{p . e v_{d} . r}=\operatorname{Dom} e v_{d} \cdot r_{A_{T}} \circ \alpha_{p}$ and $\alpha_{p . e v_{d} \cdot r}=e v_{d} \cdot r_{A_{T}} \circ \alpha_{p}$.

CASE $S=S_{1}+S_{2}, q=\varepsilon_{j} . r$ : From the induction hypothesis we assume Dom $\alpha_{p . \varepsilon_{j} . r}=\operatorname{Dom} r_{A_{T}} \circ \alpha_{p . \varepsilon_{j}}$ and $\alpha_{p . \varepsilon_{j} . r}=r_{A_{T}} \circ \alpha_{p \cdot \varepsilon_{j}}$. We know $x \in \operatorname{Dom} \alpha_{p}$ iff $(p) \downarrow \in x$ and $x \in \operatorname{Dom} \alpha_{p . \varepsilon_{j}}$ iff $\left(p . \varepsilon_{j}\right) \downarrow \in x$. Also, $(p) \downarrow \in x$ implies either $\left(p \cdot \varepsilon_{1}\right) \downarrow \in x$ or $\left(p . \varepsilon_{2}\right) \downarrow \in x$ and $\left(p . \varepsilon_{j}\right) \downarrow \in x$ implies $\alpha_{p}(x)=\iota_{j} \alpha_{p . \varepsilon_{j}}(x)$. Therefore Dom $\alpha_{p . \varepsilon_{j}}=\operatorname{Dom} \varepsilon_{j} \circ \alpha_{p}$ and $\alpha_{p . \varepsilon_{j}}=\varepsilon_{j} \circ \alpha_{p}$. Hence, since $\left(\varepsilon_{j} \cdot r\right)_{A_{T}}=r_{A_{T}} \circ \varepsilon_{j}, \operatorname{Dom} \alpha_{p \cdot \varepsilon_{j} \cdot r}=\operatorname{Dom} \varepsilon_{j} \cdot r_{A_{T}} \circ \alpha_{p}$ and $\alpha_{p \cdot \varepsilon_{j} \cdot r}=\varepsilon_{j} \cdot r_{A_{T}} \circ \alpha_{p}$. 
Definition 5.9 Let $\alpha_{T}=\alpha_{\langle\rangle}$, i.e. $\alpha_{p}$ for $T \stackrel{\langle\rangle}{\leadsto} T$.

COROLlary 5.10

(1) $\operatorname{Dom} \alpha_{T}=A_{T}$

(2) For every path $T \stackrel{q}{\rightsquigarrow} U$, Dom $\alpha_{q}=\left\{x \mid \alpha_{T}(x) \in \operatorname{Dom} q_{A_{T}}\right\}$ and $\alpha_{q}=q_{A_{T}} \circ \alpha_{T}$.

\section{Proof}

(1) Every maximally $\perp$-free $T \mathcal{R}_{T}^{+}$-theory contains axiom $2,(\langle\rangle) \downarrow$, so $A_{T}=$ Dom $\alpha_{T}$.

(2) Let $p=\langle\rangle$ in Lemma 5.8 .

An important property of the canonical coalgebra is that each state $x$ satisfies all and only the wffs that it contains.

LEMMA 5.11 (TRUTH LEMMA) $\alpha_{T}, x \vDash \varphi$ iff $\varphi \in x$.

Proof By induction on the structure of $\varphi$ :

$\operatorname{CASE} \varphi=\perp: \alpha_{T}, x \not \neq \perp$ and $\perp \notin x$ by definition.

CASE $\varphi=(p) \downarrow: \quad \alpha_{T}, x \models(p) \downarrow$

iff $\quad \alpha_{T}(x) \in \operatorname{Dom} p_{A_{T}} \quad$ by the semantics of $(p) \downarrow$

iff $x \in \operatorname{Dom} \alpha_{p} \quad$ by Corollary 5.10

iff $(p) \downarrow \in x \quad$ by the definition of $\alpha_{p}$

CASE $\varphi=(p) d:(\Rightarrow)$ If $\alpha_{T}, x \vDash(p) d$ then $\alpha_{T}, x \vDash(p) \downarrow$ and $p_{A_{T}}\left(\alpha_{T}(x)\right)=d$.

Therefore $(p) \downarrow \in x$, by the previous CASE, and $\alpha_{p}(x)=d$, by Corollary 5.10. Hence, by Lemma 5.5 and the definition of $\alpha_{p}$ (Lemma 5.8), $(p) d \in x$.

$(\Leftarrow)$ If $(p) d \in x$ then $(p) \downarrow \in x$ by axiom 7. By its definition (Lemma 5.8) $\alpha_{p}(x)=d$ and thus $p_{A_{T}}\left(\alpha_{T}(x)\right)=d$, by Corollary 5.10. Since $\alpha_{T}, x \vDash(p) \downarrow$ by the previous CASE it follows that $\alpha_{T}, x \models(p) d$.

Now assume the lemma holds for $\theta$ and $\psi$. 
CASE $\varphi=\theta \rightarrow \psi:$

$\begin{array}{ll} & \alpha_{T}, x \models \theta \rightarrow \psi \\ \text { iff } \quad \alpha_{T}, x \models \theta \text { implies } \alpha_{T}, x \models \psi & \text { by the semantics of } \rightarrow \\ \text { iff } \theta \in x \text { implies } \psi \in x & \text { by the induction hypothesis } \\ \text { iff } \theta \rightarrow \psi \in x & \text { by Detachment and the } \\ & \text { tautology } \theta \rightarrow(\psi \rightarrow(\theta \rightarrow \psi)) \in x\end{array}$

CASE $\varphi=[p] \psi:(\Rightarrow)$ Suppose $\alpha_{T}, x \vDash[p] \psi$. If $\alpha_{T}, x \not \models(p) \downarrow$ then $(p) \uparrow \in x$. From axioms 10, 1 and 11, $(p) \uparrow \rightarrow[p] \perp,[p](\perp \rightarrow \psi),[p](\perp \rightarrow \psi) \rightarrow([p] \perp \rightarrow$ $[p] \psi) \in x$, and so by Detachment it follows that $[p] \psi \in x$.

Otherwise, $\alpha_{T}, x \vDash(p) \downarrow$ and $\alpha_{T}, p_{A_{T}}\left(\alpha_{T}(x)\right) \vDash \psi$. Therefore $(p) \downarrow \in x$ and $\alpha_{T}, x_{p} \vDash \psi$, as $p_{A_{T}}\left(\alpha_{T}(x)\right)=\alpha_{p}(x)=x_{p}$ by Lemma 5.8 and Corollary 5.10 . Hence $\psi \in x_{p}$ by the induction hypothesis and $[p] \psi \in x$.

$(\Leftarrow)$ Suppose $[p] \psi \in x$. By negation completeness either $(p) \uparrow \in x$ or $(p) \downarrow \in x$. For the former it immediately follows that $\alpha_{T}, x \not \models(p) \downarrow$ and therefore $\alpha_{T}, x \models[p] \psi$. For the latter $\alpha_{T}, x \models(p) \downarrow$ and $\psi \in x_{p} \in A_{T}$ so, by the induction hypothesis, $\alpha_{T}, x_{p} \vDash \psi$. But $x_{p}=p_{A_{T}}\left(\alpha_{T}(x)\right)$; hence $\alpha_{T}, x \vDash[p] \psi$.

Now we are able to show that the canonical $T$-coalgebra is a final $T$-coalgebra.

THEOREM $5.12\left(A_{T}, \alpha_{T}\right)$ is a final T-coalgebra.

Proof Let $\langle B, \beta\rangle$ be any $T$-coalgebra. Define

$$
!_{\beta}: B \longrightarrow A_{T}: b \longmapsto\{\varphi \mid \beta, b=\varphi\}
$$

Now we show that $!_{\beta}$ is the unique morphism from $\beta$ to $\alpha_{T}$.

- $!_{\beta}(b) \in A_{T}: !_{\beta}(b)$ is a truth set, so is in $A_{T}$ by Lemma 5.4 .

- $!_{\beta}$ is a morphism: by Corollary 2.38 we need to show $p_{B}(\beta(b))=$ $p_{A_{T}}\left(\alpha_{T}\left(!_{\beta}(b)\right)\right)$ for observation paths and $!_{\beta}\left(p_{B}(\beta(b))\right)=p_{A_{T}}\left(\alpha_{T}\left(!_{\beta}(b)\right)\right)$ for state paths. 
Firstly, $\quad \beta(b) \in \operatorname{Dom} p_{B}$
iff $\beta, b \vDash(p) \downarrow$
by the semantics of $(p) \downarrow$
iff $(p) \downarrow \in !_{\beta}(b)$
by the definition of $!_{\beta}$
iff $\alpha_{T}, !_{\beta}(b) \vDash(p) \downarrow$
by the Truth Lemma (5.11)
iff $\alpha_{T}\left(!_{\beta}(b)\right) \in \operatorname{Dom} p_{A_{T}}$
by the semantics of $(p) \downarrow$

For observation paths, when $\beta, b=(p) \downarrow$ :

$$
\begin{aligned}
& p_{B}(\beta(b))=c \\
& \text { iff } \beta, b \vDash(p) c \quad \text { by the semantics of }(p) c \\
& \text { iff }(p) c \in !_{\beta}(b) \quad \text { by the definition of } !_{\beta} \\
& \text { iff } \alpha_{T}, !_{\beta}(b) \vDash(p) c \quad \text { by the Truth Lemma (5.11) } \\
& \text { iff } p_{A_{T}}\left(\alpha_{T}\left(!_{\beta}(b)\right)\right)=c \quad \text { by the semantics of }(p) c
\end{aligned}
$$

For state paths, when $\beta, b \models(p) \downarrow$ :

$$
\begin{array}{lll} 
& \varphi \in !_{\beta}\left(p_{B}(\beta(b))\right) & \\
\text { iff } & \beta, p_{B}(\beta(b)) \vDash \varphi & \text { by the definition of } !_{\beta} \\
\text { iff } & \beta, b \models[p] \varphi & \text { by the semantics of }[p] \varphi \\
\text { iff } & {[p] \varphi \in !_{\beta}(b)} & \text { by the definition of } !_{\beta} \\
\text { iff } & \varphi \in p_{A_{T}}\left(\alpha_{T}\left(!_{\beta}(b)\right)\right) & \text { by the definition of } \alpha_{T}
\end{array}
$$

i.e. $!_{\beta}\left(p_{B}(\beta(b))\right)=p_{A_{T}}\left(\alpha_{T}\left(!_{\beta}(b)\right)\right)$.

Hence, $!_{\beta}$ is a $T$-morphism.

- uniqueness of $!_{\beta}$ : Let $f: B \longrightarrow A_{T}$ be a morphism from $\beta$ to $\alpha_{T}$. Now we need to show $\varphi \in f(b)$ iff $\varphi \in !_{\beta}(b)$.

$$
\begin{aligned}
& \varphi \in !_{\beta}(b) \\
& \text { iff } \beta, b=\varphi \quad \text { by the definition of } !_{\beta} \\
& \text { iff } \alpha_{T}, f(b) \vDash \varphi \quad \text { by Lemma } 3.5 \\
& \text { iff } \varphi \in f(b) \quad \text { by the Truth Lemma (5.11) }
\end{aligned}
$$

As can be seen, $\left\langle A_{T}, \alpha_{T}\right\rangle$ is a natural construction of a final coalgebra for any polynomial functor $T$, providing a proof of Theorem 2.28 . 


\section{Chapter 6}

\section{Proof Theory}

Now we will develop a proof theoretical relation $\vdash_{T}$. It will be seen that this relation is both sound (Theorem 6.4) and complete (Theorem 6.14) with respect to the semantic model. For the latter we require $\Phi_{T}$ to be countable, so we restrict $T$ to those functors constructed using countable constant sets (c.f. Lemma 3.2). The approach used to show completeness is called a "Henkin proof", which uses a canonical model (coalgebra), as constructed in the previous chapter. This technique was first introduced by Henkin [12] for first order logic and has been described abstractly for modal logics by Goldblatt [7, 8].

First, we need to extend the relation $\mathcal{R}_{T}$ further, as the relation $\mathcal{R}_{T}^{+}$used in the previous chapters is not strong enough to define $\vdash_{T}$. The new relation, which we call $R_{T}^{+}$, could have been defined and used in Chapter 5 to construct the canonical $T$-coalgebra instead of $\mathcal{R}_{T}^{+}$. Doing so would slightly simplify the thesis as a whole, but would have introduced redundancies in the chapter as a self-contained final $T$-coalgebra construction and proof of Theorem 2.28 .

Definition 6.1 The relation $\mathcal{R}_{T}^{+} \subseteq 2^{\Phi_{T}} \times \Phi_{T}$ extends $\mathcal{R}_{T}^{+}$with the addition of an implication property. It is defined as the smallest relation extending

$$
\mathcal{R}_{T}=\{\langle\{\neg(p) d: d \in D\},(p) \uparrow\rangle \mid p \text { is an observation path } T \stackrel{p}{\sim} \bar{D}\}
$$


such that

- if $\langle\Sigma, \varphi\rangle \in \mathcal{R}_{T}^{+}$then $\langle[q] \Sigma,[q] \varphi\rangle \in \mathcal{R}_{T}^{+}$for every state path $q$

- if $\langle\Sigma, \varphi\rangle \in \mathcal{R}_{T}^{+}$then $\langle\psi \rightarrow \Sigma, \psi \rightarrow \varphi\rangle \in \mathcal{R}_{T}^{+}$for every $\psi \in \Phi_{T}$

where $[q] \Sigma=\{[q] \psi \mid \psi \in \Sigma\}$ and $\psi \rightarrow \Gamma=\{\psi \rightarrow \theta \mid \theta \in \Gamma\}$.

A set $\Gamma \subseteq \Phi_{T}$ is implication rich if it is closed under $\mathcal{R}_{T}^{+}$.

The properties shown for $\mathcal{R}_{T}^{+}$and $T \mathcal{R}_{T}^{+}$-theories can be extended to apply to $\mathcal{R}_{T}^{+}$and $T \mathcal{R}_{T}^{+}$-theories as well. In particular we want to extend Lemma 4.4, Theorem 4.5 and Lemma 5.6.

LEMMA 6.2

(1) If all of the constant sets used in the construction of $T$ are countable then $\mathcal{R}_{T}^{+}$is countable.

(2) If $\Sigma \mathcal{R}_{T}^{+} \varphi$ then $\left.\Sigma\right|_{\bar{T}} \varphi$.

(3) For every state path $p$, if $\Delta$ is a $T R_{T}^{+}$-theory and $(p) \downarrow \in \Delta$ then $\Delta_{p}=$ $\{\varphi \mid[p] \varphi \in \Delta\}$ is also a $T \mathcal{R}_{T}^{+}$-theory.

\section{Proof}

(1) We define a sequence of relations $R_{i} \subseteq 2^{\Phi_{T}} \times \Phi_{T}$, where $R_{0}=R_{T}$ and $R_{n+1}$ is the result of adding to $R_{n}$ all pairs of the form $\langle[q] \Sigma,[q] \varphi\rangle$ and $\langle\psi \rightarrow \Sigma, \psi \rightarrow \varphi\rangle$, where $\langle\Sigma, \varphi\rangle \in R_{n}, \psi \in \Phi_{T}$ and $q$ is a state path from $T . R_{T}^{+}=\bigcup_{i \geqslant 0} R_{i}$ as it is the smallest relation satisfying the properties in Definition 6.1.

If $R_{n}$ is countable then the set of pairs of the form $\langle[q] \Sigma,[q] \varphi\rangle$ is countable, by Lemma 2.32, and the set of pairs of the form $\langle\psi \rightarrow \Sigma, \psi \rightarrow \varphi\rangle$ is countable, by Lemma 3.2. Thus $R_{n+1}$ is countable too.

But $\mathcal{R}_{T}$ is countable, by Lemma 4.4 , so $\mathcal{R}_{T}^{+}$is a countable union of countable sets, and hence is countable. 
(2) Recall, from the proof of Theorem 4.5, that $\mathcal{R}_{T} \subseteq \models_{\bar{T}}$ and that $\langle\Gamma, \varphi\rangle \in \models_{T}$ implies $\left.\langle[p] \Gamma,[p] \varphi\rangle \in\right|_{\bar{T}}$. Furthermore, suppose that $\left.\Gamma\right|_{\bar{T}} \varphi$ and that $\alpha,\left.x\right|_{\bar{T}} \psi \rightarrow \Gamma$, for some $\psi \in \Phi_{T}, T$-coalgebra $\alpha$ and state $x \in \operatorname{Dom} \alpha$. Then

$\begin{array}{lll} & \alpha,\left.x\right|_{\bar{T}} \psi & \\ \text { implies } & \alpha,\left.x\right|_{\bar{T}} \Gamma & \text { by the semantics of } \rightarrow \\ \text { implies } & \alpha,\left.x\right|_{\bar{T}} \varphi & \text { as }\left.\Gamma\right|_{\bar{T}} \varphi \\ \text { hence } & \alpha,\left.x\right|_{\bar{T}} \psi \rightarrow \varphi & \text { by the semantics of } \rightarrow\end{array}$

Thus $\left.\langle\Gamma, \varphi\rangle \in\right|_{\bar{T}}$ implies $\left.\langle\psi \rightarrow \Gamma, \psi \rightarrow \varphi\rangle \in\right|_{\bar{T}} . R_{T}^{+}$is the smallest relation to satisfy these three properties, hence $\Sigma \mathcal{R}_{T}^{+} \varphi$ implies $\left.\Sigma\right|_{\bar{T}} \varphi$.

(3) As for Lemma 5.6 .

We define the proof relation $\vdash_{T}$ with respect to $T R_{T}^{+}$-theories, which are purely syntactic constructs.

Definition 6.3 $\Gamma \vdash_{T} \varphi$ iff $\varphi \in \bigcap\left\{\Delta \mid \Gamma \subseteq \Delta\right.$ and $\Delta$ is a $T R_{T}^{+-}$-theory $\}$i.e. $\varphi$ belongs to every $T \mathcal{R}_{T}^{\#}$-theory extending $\Gamma$.

THEOREM 6.4 (SOUNDNESS) If $\Gamma \vdash_{T} \varphi$ then $\left.\Gamma\right|_{\bar{T}} \varphi$.

Proof Suppose $\Gamma \vdash_{T} \varphi$ and $\alpha, x \models \Gamma$ for some $T$-coalgebra $\langle A, \alpha\rangle$ and $x \in A$. We need to show $\alpha, x \vDash \varphi$, so let $\Delta=\{\psi \mid \alpha, x \vDash \psi\}$.

$\Delta$ is a $T \mathcal{R}_{T}^{+}$-theory - it contains all axioms and is closed under $\mathcal{R}_{T}^{+}$by Theorem 4.5 and Lemma 6.2 and it is closed under Detachment by the semantic definition of $\rightarrow$. Therefore, since $\Gamma \subseteq \Delta$ and $\Gamma \vdash_{T} \varphi, \varphi \in \Delta$. Hence $\alpha, x \vDash \varphi$.

There are a number of properties that we would like $\vdash_{T}$ to have.

LEMMA $6.5 \vdash_{T}$ satisfies:

(1) Closure Theorem (CT): If $\Gamma \vdash_{T} \psi$ for all $\psi \in \Delta$ and $\left.\Delta\right|_{T} \varphi$ then $\left.\Gamma\right|_{T} \varphi$

(2) Deduction Theorem (DT): $\Gamma \cup\{\varphi\} \vdash_{T} \psi$ implies $\Gamma \vdash_{T} \varphi \rightarrow \psi$ 
(3) Monotonicity: If $\Gamma \vdash_{T} \varphi$ and $\Gamma \subseteq \Delta$ then $\Delta \vdash_{T} \varphi$

(4) Detachment: If $\Gamma \vdash_{T} \varphi$ and $\Gamma \vdash_{T} \varphi \rightarrow \psi$ then $\Gamma \vdash_{T} \psi$

(5) If $\Gamma \vdash_{T} \varphi$ and $\Gamma \cup\{\varphi\} \vdash_{T} \perp$ then $\Gamma \vdash_{T} \perp$

(6) If $\Gamma \cup\{\neg \varphi\} \vdash_{T} \perp$ then $\Gamma \vdash_{T} \varphi$

(7) Box Rule (BR): If $\Gamma \vdash_{T} \varphi$ then $[p] \Gamma \vdash_{T}[p] \varphi$ for all state paths $p$

(8) Implication Rule (IR): If $\Gamma \vdash_{T} \varphi$ then $\psi \rightarrow \Gamma \vdash_{T} \psi \rightarrow \varphi$

\section{Proof}

(1) If $\Gamma \vdash_{T} \psi$ for all $\psi \in \Delta$ and $\Delta \vdash_{T} \varphi$ then any $T \mathcal{R}_{T}^{+}$-theory containing $\Gamma$ will also contain $\Delta$ and therefore $\varphi$. Hence $\Gamma \vdash_{T} \varphi$

(2) Suppose $\Gamma \cup\{\varphi\} \vdash_{T} \psi$. Let $\Delta=\left\{\chi \mid \Gamma \vdash_{T} \varphi \rightarrow \chi\right\}$. We want to show $\psi \in \Delta$, and can do so by showing that $\Delta$ is a $T \mathcal{R}_{T}^{+}$-theory containing $\Gamma \cup\{\varphi\}$.

$\theta \rightarrow(\varphi \rightarrow \theta)$ is a tautology (axiom 1) so if $\theta \in \Gamma$ or $\theta \in \Sigma_{T}$ then $\Gamma{ }_{T} \varphi \rightarrow \theta . \varphi \rightarrow \varphi$ is also a tautology so $\Gamma{ }_{T} \varphi \rightarrow \varphi$. Hence $\Sigma_{T} \cup \Gamma \cup\{\varphi\} \subseteq \Delta$. To show $\Delta$ closed under Detachment, suppose $\theta, \theta \rightarrow \chi \in \Delta$. Then from the tautology $(\varphi \rightarrow \theta) \rightarrow((\varphi \rightarrow(\theta \rightarrow \chi)) \rightarrow(\varphi \rightarrow \chi))$ it follows that $\Gamma \vdash_{T} \varphi \rightarrow \chi$.

To show $\Delta$ is implication rich, let $\langle\Sigma, \chi\rangle \in \mathcal{R}_{T}^{+}$and suppose $\Sigma \subseteq \Delta$. Then $\Gamma \vdash_{T} \varphi \rightarrow \theta$ for every $\theta \in \Sigma$. So, any $T \mathcal{R}_{T}^{+}$-theory that contains $\Gamma$ will also contain $\varphi \rightarrow \Sigma$. But $\langle\varphi \rightarrow \Sigma, \varphi \rightarrow \chi\rangle \in \mathcal{R}_{T}^{+}$so every $T \mathcal{R}_{T}^{+}$theory that contains $\Gamma$ will also contain $\varphi \rightarrow \chi$. Therefore $\chi \in \Delta$.

Since $\Delta$ is a $T R_{T}^{+}$-theory containing $\Gamma \cup\{\varphi\}$ it follows that $\Gamma \cup\{\varphi\} \vdash_{T} \psi$ implies $\Gamma \vdash_{T} \varphi \rightarrow \psi$.

(3) If $\Gamma \vdash_{T} \varphi$ and $\Gamma \subseteq \Delta$ then $\Delta \vdash_{T} \psi$ for all $\psi \in \Gamma$. Therefore, by $C T, \Delta \vdash_{T} \varphi$.

(4) $\{\varphi, \varphi \rightarrow \psi\} \vdash_{T} \psi$ so if $\Gamma \vdash_{T} \varphi$ and $\Gamma \vdash_{T} \varphi \rightarrow \psi$ then $\Gamma \vdash_{T} \psi$ follows from CT.

(5) If $\Gamma \vdash_{T} \varphi$ and $\Gamma \cup\{\varphi\} \vdash_{T} \perp$ then $\Gamma \vdash_{T} \varphi \rightarrow \perp$ by DT. So $\Gamma \vdash_{T} \perp$ by (4). 
(6) If $\left.\Gamma \cup\{\neg \varphi\}\right|_{T} \perp$ then $\left.\Gamma\right|_{T} \neg \varphi \rightarrow \perp$ by DT. By tautology $\Gamma \vdash_{T}(\neg \varphi \rightarrow \perp) \rightarrow \varphi$. Hence, by $\{\neg \varphi \rightarrow \perp,(\neg \varphi \rightarrow \perp) \rightarrow \varphi\} \vdash_{T} \varphi$ and $\mathrm{CT}, \Gamma \vdash_{T} \varphi$.

(7) Suppose $\Gamma \vdash_{T} \varphi$. To show $[p] \Gamma \vdash_{T}[p] \varphi$ let $\Delta \supseteq[p] \Gamma$ be a $T \mathcal{R}_{T}^{+}$-theory and define $\Delta_{p}=\{\psi \mid[p] \psi \in \Delta\}$. We want to show $\varphi \in \Delta_{p}-$ it is sufficient to show that $\Delta_{p}$ is a $T \mathcal{R}_{T}^{+}$-theory containing $\Gamma$. But $\Gamma \subseteq \Delta_{p}$, by definition, and $D_{p}$ is a $T R_{T}^{++}$-theory, by Lemma 5.6 .

(8) This is a general proof that IR follows from CT, DT and propositional inference.

Suppose $\Gamma \vdash_{T} \varphi$. Since $(\psi \rightarrow \Gamma) \cup\{\psi\} \vdash_{T} \theta$ for all $\theta \in \Gamma$ it follows that $(\psi \rightarrow \Gamma) \cup\{\psi\} \vdash_{T} \varphi$ by CT. Therefore $\psi \rightarrow \Gamma \vdash_{T} \psi \rightarrow \varphi$, by DT.

One of the most important concepts associated with $\vdash_{T}$ is the notion of consistency. It is important to recognise when a set of wff can be used to prove something that is not true (e.g. $\perp$ ) and is thus inconsistent.

DEFINITION 6.6 $\Gamma$ is $\vdash_{T}$-inconsistent if $\Gamma \vdash_{T} \perp$ and $\vdash_{T}$-consistent otherwise. $\Gamma$ is finitely $\vdash_{T}$-consistent if all finite subsets of $\Gamma$ are $\vdash_{T}$-consistent. $\Gamma$ is a maximally $\vdash_{T}^{-}$-consistent $T \mathcal{R}_{T}^{+}$-theory if it is a negation complete and $\vdash_{T}$-consistent $T \mathcal{R}_{T}^{+}$ theory.

The use of the word 'maximal' in the previous definition is justified by the following lemma.

LEMMA 6.7 Suppose $\Gamma$ and $\Delta$ are $\vdash_{T}$-consistent sets, $\Gamma$ is negation complete and $\Gamma \subseteq \Delta$. Then $\Gamma=\Delta$.

Proof As for Lemma 5.3, with Lemma 6.8(3).

LEMMA 6.8

(1) $T R_{T}^{+}$-theories are $\vdash_{T}$-closed, i.e. if $\Delta$ is a $T R_{T}^{+}$-theory and $\Delta \vdash_{T} \varphi$ then $\varphi \in \Delta$

(2) If $\perp \in \Gamma$ then $\Gamma$ is $\vdash_{T}$-inconsistent. 
(3) If $\Delta$ is a $T R_{T}^{+}$-theory then it is $\vdash_{T}^{-}$consistent iff $\perp \notin \Delta$.

\section{Proof}

(1) Suppose $\Delta$ is a $T \mathcal{R}_{T}^{+}$-theory, $\Gamma \vdash_{T} \varphi$ and $\Gamma \subseteq \Delta$. By definition $\varphi$ is a member of every $T R_{T}^{+}$-theory that contains $\Delta$, hence $\varphi \in \Delta$.

(2) If $\perp \in \Gamma$ then $\perp$ will be a member of every $T \mathcal{R}_{T}^{\#}$-theory that contains $\Gamma$. Therefore $\Gamma \vdash_{T} \perp$.

(3) The $(\Rightarrow)$ direction follows from the contraposition of (2) above. For $(\Leftarrow)$ assume that $\Delta$ is a $T R_{T}^{+}$-theory and $\perp \notin \Delta$. It follows that $\perp \notin\left\{\Delta^{\prime} \mid\right.$ $\Delta^{\prime}$ is a $T R_{T}^{+}$-theory and $\left.\Delta \subseteq \Delta^{\prime}\right\}$, hence by definition $\left.\Delta\right|_{T} \perp$.

LEMMA 6.9 All truth sets are maximally $\left.\right|_{T}$-consistent $T \mathcal{R}_{T}^{+}$-theories.

Proof As for Lemma 5.4 $\{\varphi \mid \alpha, x \models \varphi\}$ is a $T R_{T}^{+}$-theory by Theorem 4.5 . Lemma 6.2(1) and the semantics of $\rightarrow$. It is negation complete by Remark 3.4 and is $\vdash_{T}$-consistent by Lemma 6.8(3).

At this point in a Henkin proof we would normally construct a canonical model (coalgebra) using the proof relation - in this case using maximally $\vdash_{T}$-consistent $T \mathcal{R}_{T}^{+}$-theories. However we have already constructed a canonical coalgebra, using maximally $\perp$-free $T \mathcal{R}_{T}^{+}$-theories. These two notions are actually the same and we can use the existing canonical coalgebra with the alternate definition $A_{T}=\left\{x \mid x\right.$ is a maximally $\vdash_{T}$-consistent $T \mathcal{R}_{T}^{+}$theory\}.

LEMMA 6.10 A set of wffs is a maximally $\perp$-free $T R_{T}^{+}$-theory iff it is a maximally $\vdash_{T}$-consistent $T R_{T}^{+}$-theory.

Proof $\quad(\Leftarrow)$ Every $T \mathcal{R}_{T}^{+}$-theory is a $T \mathcal{R}_{T}^{+}$-theory as $\mathcal{R}_{T}^{+} \supseteq \mathcal{R}_{T}^{+}$. So Lemma 6.8(3) gives that every maximally $\vdash_{T}$-consistent $T \mathcal{R}_{T}^{+}$-theory is a maximally $\perp$-free $T \mathcal{R}_{T}^{+}$-theory.

$(\Rightarrow)$ If $\Delta$ is a maximally $\perp$-free $T \mathcal{R}_{T}^{+}$-theory then it is a member of $A_{T}$ and, by the Truth Lemma (5.11), $\alpha_{T}, \Delta \vDash \varphi$ iff $\varphi \in \Delta$. Thus $\Delta$ is a truth set and hence, by Lemma 6.9 , is a maximally $\vdash_{T}$-consistent $T \mathcal{R}_{T}^{+}$-theory. 


\section{LEMMA 6.11}

(1) If $\Gamma$ is finitely $\vdash_{T}$-consistent then so is one of $\Gamma \cup\{\varphi\}$ and $\Gamma \cup\{\neg \varphi\}$ for all $\varphi$.

(2) If $\Gamma$ is negation complete and finitely $\vdash_{T}$-consistent then $\Gamma$ is closed under Detachment and contains all axioms.

(3) If $\Gamma$ is finitely $\vdash_{T}$-consistent then $\Gamma$ is maximally finitely $\vdash_{T}$-consistent iff it is negation complete.

\section{Proof}

(1) If the conclusion is false then $\left.\Gamma_{0} \cup\{\varphi\}\right|_{T} \perp$ and $\left.\Gamma_{1} \cup\{\neg \varphi\}\right|_{T} \perp$ for some $\varphi$ and some $\Gamma_{0}, \Gamma_{1} \subseteq \Gamma$. Then, by DT and monotonicity, $\Gamma_{0} \cup \Gamma_{1} \vdash_{T} \varphi \rightarrow \perp$ and $\Gamma_{0} \cup \Gamma_{1} \vdash_{T} \neg \varphi \rightarrow \perp$.

But $\{\varphi \rightarrow \perp, \neg \varphi \rightarrow \perp\} \vdash_{T} \perp$ as every $T \mathcal{R}_{T}^{+}$-theory that contains $\varphi \rightarrow \perp$ and $\neg \varphi \rightarrow \perp=(\varphi \rightarrow \perp) \rightarrow \perp$ also contains $\perp$, by Detachment. Therefore, by CT, $\Gamma_{0} \cup \Gamma_{1} \vdash_{T} \perp$.

Therefore $\Gamma$ is not finitely $\vdash_{T}$-consistent.

(2) Let $\Gamma$ be negation complete and finitely $\vdash_{T}$-consistent.

$\Gamma$ is closed under Detachment as if $\varphi, \varphi \rightarrow \psi \in \Gamma$ and $\psi \notin \Gamma$ then $\neg \psi \in \Gamma$, so $\Gamma$ would contain the finite $\vdash_{T}$-inconsistent set $\{\varphi, \varphi \rightarrow \psi, \neg \psi\}$.

If $\varphi$ is an axiom then $\{\neg \varphi\}$ is a finite $\vdash_{T}$-inconsistent set, so $\varphi \in \Gamma$ by negation completeness.

(3) Let $\Gamma$ be maximally finitely $\vdash_{T}$-consistent. Then for all $\varphi$ by (1) either $\Gamma \cup\{\varphi\}=\Gamma$ or $\Gamma \cup\{\neg \varphi\}=\Gamma$. Therefore $\Gamma$ is negation complete.

Conversely, if $\Gamma$ is finitely $\vdash_{T}$-consistent and negation complete let $\Gamma \subset$ $\Delta$. Then there exists $\varphi \in \Delta$ with $\neg \varphi \in \Gamma$, so $\Delta$ contains the finitely $\vdash_{T}$-inconsistent set $\{\varphi, \neg \varphi\}$. Therefore $\Gamma$ has no finitely $\vdash_{T}$-consistent extension and is maximally finitely $\vdash_{T}$-consistent. 
COROLLARY 6.12 If $\Gamma$ is maximally finitely $\vdash_{T}$-consistent and implication rich then $\Gamma$ is a maximally $\vdash_{T}$-consistent $T R_{T}^{*}$-theory.

Proof If $\Gamma$ is maximally finitely $\vdash_{T}$-consistent and implication rich then by (2) and (3) of Lemma 6.11 it is also negation complete, closed under Detachment and contains all axioms, and therefore a $T R_{T}^{+*}$-theory.

If $\Gamma$ was $\vdash_{T}$-inconsistent, i.e. $\Gamma \vdash_{T} \perp$, then $\{\perp\} \subseteq \Gamma$, by Lemma 6.8 (3), contrary to the fact that it is finitely $\vdash_{T}-$-consistent. Hence it is a maximally $\vdash_{T}$-consistent $T R_{T}^{+}$-theory.

LEMMA 6.13 (EXTENSION LEMMA) Every $\vdash_{T}$-consistent set can be extended to a maximally $\vdash_{T}$-consistent $T R_{T}^{+}$-theory.

Proof Suppose $\Gamma$ is $\vdash_{T}-$ consistent. Recall that $\Phi_{T}$ and $\mathcal{R}_{T}^{+}$are countable, by Lemma 3.2 and Lemma 6.2(1), using the assumption for this chapter that no uncountable constant sets are used in the construction of $T$. Fix an enumeration $\varphi_{0}, \varphi_{1}, \ldots, \varphi_{n}, \ldots$ of $\Phi_{T}$ and an enumeration $\left\langle\Sigma_{0}, \psi_{0}\right\rangle,\left\langle\Sigma_{1}, \psi_{1}\right\rangle, \ldots$, $\left\langle\Sigma_{n}, \psi_{n}\right\rangle, \ldots$ of $\mathcal{R}_{T}^{+}$.

Let $\Delta_{0}=\Gamma$. Assume $\Delta_{n}$ is defined and $\vdash_{T}$-consistent. If $\Delta_{n} \vdash_{T} \varphi_{n}$ then let

$$
\Delta_{n+1}=\Delta_{n} \cup\left\{\varphi_{n}\right\}
$$

which is $\vdash_{T}$-consistent by Lemma 6.55). Alternatively, $\Delta_{n} H_{T} \varphi_{n}$. If $\varphi_{n} \neq \psi_{m}$ for every $m$, then let

$$
\Delta_{n+1}=\Delta_{n} \cup\left\{\neg \varphi_{n}\right\}
$$

which is $\vdash_{T}$-consistent by Lemma 6.5 6). Otherwise there is an $m$ such that $\psi_{m}=\varphi_{n}$. If $\left.\Delta_{n} \cup\left\{\neg \varphi_{n}\right\}\right|_{T} \psi_{m}$ then $\left.\Delta_{n}\right|_{T} \varphi_{n}$ - a contradiction - so $\left.\Delta_{n} \cup\left\{\neg \varphi_{n}\right\}\right|_{T} \psi_{m}$. Therefore, since $\Sigma_{m} \vdash_{T} \psi_{m}$, by CT there exists a $\theta \in \Sigma_{m}$ such that $\left.\Delta_{n} \cup\left\{\neg \varphi_{n}\right\}\right|_{T} \theta$. Let

$$
\Delta_{n+1}=\Delta_{n} \cup\left\{\neg \varphi_{n}, \neg \theta\right\}
$$

which is $\vdash_{T}$-consistent by Lemma 6.5)(6).

Now let $\Delta=\bigcup_{i \geqslant 0} \Delta_{i}$. 
$\Delta$ is finitely $\vdash_{T}$-consistent - all finite subsets of $\Delta$ are subsets of some $\Delta_{i}$, so if a finite subset was not $\vdash_{T}$-consistent then $\Delta_{i}{ }^{\prime}$ s $\vdash_{T}$-consistency would be violated.

$\Delta$ is negation complete by construction, so is maximally finitely $\vdash_{T}$ consistent, by Lemma 6.11(2). But it is also implication rich by construction. Hence, by Corollary 6.12, $\Delta$ is a maximally $\vdash_{T}^{-}$-consistent $T \mathcal{R}_{T}^{++}$-theory.

This now allows us to show that $\vdash_{T}$ is complete.

THEOREM 6.14 (COMPLETENESS) The following are equivalent:

(1) $\Gamma \vdash_{T} \varphi$

(2) $\Gamma \models_{\bar{T}} \varphi$

(3) $\Gamma \models^{\alpha_{T}} \varphi$

\section{Proof}

$(1) \Rightarrow(2)$ Theorem 6.4

(2) $\Rightarrow$ (3) Follows as $\alpha_{T}$ is a $T$-coalgebra.

(3) $\Rightarrow$ (1) If $\left.\Gamma\right|_{T} \varphi$ then $\Gamma \cup\{\neg \varphi\}$ is $\vdash_{T}$-consistent, by Lemma 6.5 6). Therefore, by the Extension Lemma (6.13), there exists a maximally $\left.\right|_{T}$-consistent $T \mathcal{R}_{T}^{+}$-theory $x \supseteq \Gamma \cup\{\neg \varphi\}$. By the Truth Lemma (5.11), $\alpha_{T}, x \vDash \Gamma$ and $\alpha_{T}, x \not \neq \varphi$, hence $\Gamma \|^{\alpha_{T}} \varphi$. 


\section{Bibliography}

[1] Peter Aczel and Nax Mendler. A final coalgebra theorem. In D. H. Pitt et al., editors, Category Theory and Computer Science. Proceedings 1989, volume 389 of Lecture Notes in Computer Science, pages 357-365. Springer-Verlag, 1989.

[2] Jon Barwise and Lawrence Moss. Vicious Circles: On the Mathematics of Non-wellfounded Phenomena. CSLI Lecture Notes No. 60. CSLI Publications, Stanford, California, 1996. Distributed by Cambridge University Press.

[3] Corina Cîrstea. A coequational approach to specifying behaviours. In B. Jacobs and J. Rutten, editors, Proceedings of the Second Workshop on Coalgebraic Methods in Computer Science, volume 19 of Electronic Notes in Theoretical Computer Science, pages 142-163. Elsevier, 1999.

[4] Corina CîRstea. A coalgebraic equational approach to specifying observational structures. Theoretical Computer Science, 280:35-68, 2002.

[5] Andrea Corradini. A complete calculus for equational deduction in coalgebraic specification. Technical Report SEN-R9723, Centrum voor Wiskunde en Informatica (CWI), Amsterdam, 1997.

[6] Andrea Corradini. A completeness result for equational deduction in coalgebraic specification. In Francesco Parisi Presicce, editor, 
Recent Trends in Algebraic Development Techniques, volume 1376 of Lecture Notes in Computer Science, pages 190-205. Springer-Verlag, 1998.

[7] Robert Goldblatt. An abstract setting for Henkin proofs. Topoi, 3:37-41, 1984. Reprinted as chapter 8 of [9].

[8] Robert Goldblatt. A framework for infinitary modal logic. In Mathematics of Modality [9], chapter 9, pages 213-299.

[9] Robert Goldblatt. Mathematics of Modality. Number 43 in CSLI Lecture Notes. CSLI Publications, Stanford, California, 1993. Distributed by Cambridge University Press.

[10] Robert GoldBLATt. What is the coalgebraic analogue of Birkhoff's variety theorem? Theoretical Computer Science, 266:853-886, 2001.

[11] Robert Goldblatt. Equational logic of polynomial coalgebras. In Philippe Balbiani, Nobu-Yuki Suzuki, Frank Wolter, and Michael Zakharyaschev, editors, Advances in Modal Logic, Volume 4, pages 149184. King's College Publications, King's College London, 2003.

[12] LEON HENKIN. The completeness of the first-order functional calculus. Journal of Symbolic Logic, 14:159-166, 1949.

[13] Matthew Hennessy and Robin Milner. On observing nondeterminism and concurrency. In J. W. de Bakker and J. van Leeuwen, editors, Automata, Languages and Programming. Proceedings 1980, volume 85 of Lecture Notes in Computer Science, pages 299-309. SpringerVerlag, 1980.

[14] Matthew Hennessy and Robin Milner. Algebraic laws for nondeterminism and concurrency. Journal of the Association for Computing Machinery, 32:137-161, 1985. 
[15] Claudio Hermida. Fibrations, Logical Predicates and Indeterminates. $\mathrm{PhD}$ thesis, University of Edinburgh, 1993. Technical report LFCS93-277. Also available as Aarhus University DAIMI Technical report PB-462.

[16] Claudio Hermida and Bart Jacobs. Structural induction and coinduction in a fibrational setting. Information and Computation, 145:107-152, 1998.

[17] BART JACOBS. Objects and classes, co-algebraically. In B. Freitag, C.B. Jones, C. Lengauer, and H.-J. Schek, editors, Object-Orientation with Parallelism and Persistence, pages 83-103. Kluwer Academic Publishers, 1996.

[18] BART JACOBS. Towards a duality result in coalgebraic modal logic. In H. Reichel, editor, Proceedings of the Third Workshop on Coalgebraic Methods in Computer Science, volume 33 of Electronic Notes in Theoretical Computer Science, pages 160-195. Elsevier, 2000.

[19] BART JACOBS AND ERIK POlL. Java program verification at Nijmegen: Developments and perspective. Technical Report NIIIR0318, Computing Science Institute, University of Nijmegen, 2002.

[20] Bart Jacobs AND ERIK POll. Coalgebras and monads in the semantics of Java. Theoretical Computer Science, 291:329-349, 2003.

[21] BART JaCOBS AND JAN RUtTen. A tutorial on (co)algebras and (co)induction. Bulletin of the European Association for Theoretical Computer Science, 62:222-259, 1997.

[22] AleXAnder Kurz. Logics for Coalgebras and Applications to Computer Science. PhD thesis, Ludwig-Maximilians-Universität München, 2000.

[23] Alexander KURz. Specifying coalgebras with modal logic. Theoretical Computer Science, 260:119-138, 2001. 
[24] JOACHIM LAMBEK. A fixpoint theorem for complete categories. Mathematische Zeitschrift, 103:151-161, 1968.

[25] LoOP PROJECT. http://www.cs.kun.nl/sos/research/ $100 \mathrm{p} /$.

[26] SAunders MaC LANe. Categories for the Working Mathematician. Springer-Verlag, second edition, 1998.

[27] Lawrence S. Moss. Coalgebraic logic. Annals of Pure and Applied Logic, 96:277-317, 1999. See erratum [28].

[28] LaWrence S. Moss. Erratum to "Coalgebraic Logic". Annals of Pure and Applied Logic, 99:241-259, 1999.

[29] DAVID PARK. Concurrency and automata on infinite sequences. In P. Deussen, editor, Theoretical Computer Science, volume 104 of Lecture Notes in Computer Science, pages 167-183. Springer-Verlag, 1981.

[30] Dirk Pattinson. An introduction to the theory of coalgebras. NASSLLI Lecture Notes, 2003. http://www. indiana.edu/ nasslli/datas/DirkPattinson.pdf.

[31] ERIK POLL. A coalgebraic semantics of subtyping. Theoretical Informatics and Applications, 35:61-81, 2001.

[32] HoRSt ReICHEL. An approach to object semantics based on terminal co-algebras. Mathematical Structures in Computer Science, 5:129-152, 1995.

[33] Martin Rössiger. From modal logic to terminal coalgebras. Theoretical Computer Science, 260:209-228, 2001.

[34] J. J. M. M. RutTEN. Universal coalgebra: a theory of systems. Theoretical Computer Science, 249(1):3-80, 2000. 
[35] J. J. M. M. RUtTen AND D. TURI. On the foundations of final semantics: non-standard sets, metric spaces, partial orders. In J. W. de Bakker, W.-P. de Roever, and G. Rozenberg, editors, Proc. REX Workshop on Semantics, volume 666 of Lecture Notes in Computer Science, pages 447-530. Springer, 1993. 